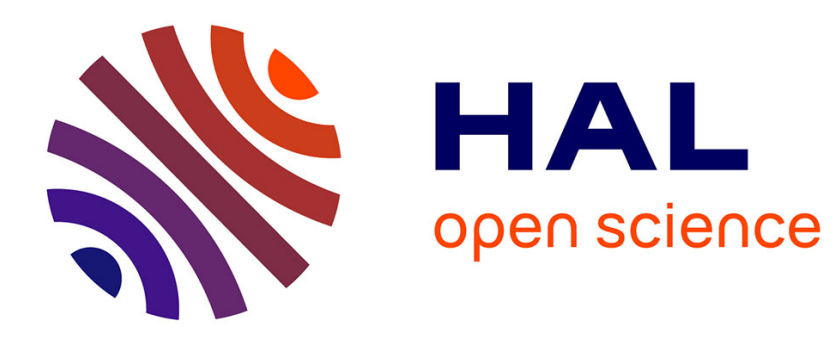

\title{
Teichmüller spaces and holomorphic dynamics
}

Xavier Buff, Guizhen Cui, Lei Tan

\section{To cite this version:}

Xavier Buff, Guizhen Cui, Lei Tan. Teichmüller spaces and holomorphic dynamics. 2011. hal00610715

\section{HAL Id: hal-00610715 \\ https://hal.science/hal-00610715}

Preprint submitted on 23 Jul 2011

HAL is a multi-disciplinary open access archive for the deposit and dissemination of scientific research documents, whether they are published or not. The documents may come from teaching and research institutions in France or abroad, or from public or private research centers.
L'archive ouverte pluridisciplinaire HAL, est destinée au dépôt et à la diffusion de documents scientifiques de niveau recherche, publiés ou non, émanant des établissements d'enseignement et de recherche français ou étrangers, des laboratoires publics ou privés. 


\title{
Teichmüller spaces and holomorphic dynamics
}

\author{
BUFF Xavier* CUI Guizhen** TAN Lei \\ Université de Toulouse, UPS, INSA, UT1, UTM \\ Institut de Mathématiques de Toulouse \\ 31062 Toulouse, France \\ email: xavier.buff@math.univ-toulouse.fr \\ Academy of Mathematics and Systems Science \\ Chinese Academy of Sciences \\ Beijing 100190, People's Republic of China \\ email: gzcui@math.ac.cn \\ Université d'Angers, Faculté des sciences, LAREMA \\ 2 Boulevard Lavoisier, 49045 Angers cedex 01, France \\ email: tanlei@math.univ-angers.fr
}

\begin{abstract}
One fundamental theorem in the theory of holomorphic dynamics is Thurston's topological characterization of postcritically finite rational maps. Its proof is a beautiful application of Teichmüller theory. In this chapter we provide a self-contained proof of a slightly generalized version of Thurston's theorem (the marked Thurston's theorem). We also mention some applications and related results, as well as the notion of deformation spaces of rational maps introduced by A. Epstein.
\end{abstract}

\section{Introduction}

Let $f(z)=p(z) / q(z)$ be a rational map with $p$ and $q$ relatively prime polynomials. The degree $d=\operatorname{deg}(f)$ of $f$ is defined to be the maximum of the degrees of $p$ and $q$. In the following we will always assume that $\operatorname{deg}(f)>1$.

The iteration of $f$ generates a holomorphic dynamical system on the Riemann sphere $\widehat{\mathbb{C}}$, and partitions the sphere into two dynamically natural subsets

\footnotetext{
*Work partially supported by the grant ANR-08-JCJC-0002, the IUF and the EU Research Training Network on Conformal Structures and Dynamics.

** Work partially supported by the grants no. 10831004 and no. 10721061 of NNSF of China, and by Hua Loo-Keng Key Laboratory of Mathematics, Chinese Academy of Sciences.

${ }^{\ddagger}$ Work partially supported by the EU Research Training Network on Conformal Structures and Dynamics, and the Pays-de-la-Loire regional grant MATPYL.
} 
$\widehat{\mathbb{C}}=\mathcal{J}_{f} \sqcup \mathcal{F}_{f}$, where by definition

$\mathcal{F}_{f}=\left\{z \in \widehat{\mathbb{C}} \mid\left\{\left.f^{\circ n}\right|_{U}\right\}_{n \geq 0}\right.$ is a normal family on some neighborhood $U$ of $\left.z\right\}$.

The set $\mathcal{J}_{f}$ (resp. $\mathcal{F}_{f}$ ) is called the Julia set (resp. the Fatou set) of $f$.

Roughly speaking, $\mathcal{F}_{f}$ consists of the set of initial values $z$ such that the long term behavior of the iterated orbit $\left(f^{\circ n}(z)\right)_{n \geq 0}$ is insensitive to small perturbations of $z$. The simplest example is given by $f(z)=z^{2}$, for which $\mathcal{F}_{f}=\{|z| \neq 1\}$ and $\mathcal{J}_{f}=\{|z|=1\}$. With a little effort one can also show that for $f(z)=z^{2}-2, \mathcal{J}_{f}=[-2,2]$. There are however very few rational maps for which the Julia set can be described by smooth equations, as $\mathcal{J}_{f}$ often presents a fractal shape.

The orbit of a point $z$ is simply $\left\{f^{\circ n}(z), n \geq 0\right\}$. We say that $z$ is periodic if there is $p$ such that $f^{p}(z)=z$. By a classical result of Fatou and Julia, there are at most finitely many periodic points outside the Julia set $\mathcal{J}_{f}$ (more precisely, all repelling periodic points are in the Julia set and there are finitely many non-repelling periodic points; see Theorem 4.3 below), and $\mathcal{J}_{f}$ is compact containing uncountably many points, in which the periodic points form a countable dense subset.

The rational map $f$ is proper and the Julia and Fatou sets are completely invariant: $f^{-1}\left(\mathcal{J}_{f}\right)=f\left(\mathcal{J}_{f}\right)=\mathcal{J}_{f}$ and $f^{-1}\left(\mathcal{F}_{f}\right)=f\left(\mathcal{F}_{f}\right)=\mathcal{F}_{f}$. As a consequence, $f$ maps each Julia (resp. Fatou) component onto another Julia (resp. Fatou) component as a proper map.

We consider $f$ as a branched covering of $\widehat{\mathbb{C}}$. With finitely many exceptions, every value $w \in \widehat{\mathbb{C}}$ has exactly $d$ preimages. More precisely, denote by $\mathcal{C}_{f}$ the set of points $z \in \widehat{\mathbb{C}}$ where $f$ is not locally injective. These points are called the critical points of $f$. Let $\mathcal{V}_{f}=f\left(\mathcal{C}_{f}\right)$ be the set of critical values of $f$. Then $f: \widehat{\mathbb{C}} \backslash f^{-1}\left(\mathcal{V}_{f}\right) \rightarrow \widehat{\mathbb{C}} \backslash \mathcal{V}_{f}$ is an (unramified) covering of degree $d$.

The postcritical set $\mathcal{P}_{f}$ of $f$ is defined to be

$$
\mathcal{P}_{f}=\operatorname{closure}\left(\bigcup_{z \in \mathcal{C}_{f}, n \geq 1}\left\{f^{\circ n}(z)\right\}\right) .
$$

In a certain sense, this set captures the essence of the dynamical system generated by $f$. We say that $f$ is postcritically finite if $\mathcal{P}_{f}$ is finite. This is equivalent to the fact that all critical points of $f$ are eventually periodic under iteration.

A rational map $f$ is hyperbolic if it is uniformly expanding near its Julia set. These are the natural analogues of Smale's Axiom A maps in this setting. If in addition the Julia set is connected, the dynamics of $f$ on $J_{f}$ is equivalent to the dynamics of a map $f_{0}$ which is postcritically finite.

We may also forget the analytic nature of a rational map and consider it as a topological (orientation preserving) branched covering of the two-sphere $S^{2}$. As the notions of degree, critical points, postcritical set and postcritical 
finiteness are topological, they are naturally defined for a branched covering as well.

In the early eighties, Thurston gave a complete topological characterization of postcritically finite rational maps (see [Th1, DH1]), which can be stated roughly as follows: The set of postcritically finite rational maps (except the Lattès examples) are in one-to-one correspondence with the homotopy classes of postcritically finite branched self-coverings of $S^{2}$ with no Thurston obstructions (see Section 2.1 for a more precise statement).

This result has then become a fundamental theorem in the theory of holomorphic dynamics, together with some surprising applications outside the field.

Teichmüller theory plays an essential role in Thurston's proof of his theorem. An outline goes as follows: To a postcritically finite branched covering $F$ of $S^{2}$ one can associate the Teichmüller space $\mathcal{T}$ of the punctured sphere $S^{2} \backslash \mathcal{P}_{F}$. The pullback of complex structures by $F$ induces a weakly contracting operator $\sigma$ on $\mathcal{T}$. The main point is to prove that in the absence of obstructions, $\sigma$ has a unique fixed point in $\mathcal{T}$. This fixed point represents a complex structure that is invariant (up to isotopy) by $F$, thus turns $F$ into an analytic branched covering, i.e. a rational map.

Therefore in order to build a rational map with desired combinatorial properties one may first construct a branched covering $F$ as a topological model (this is a lot more flexible than building holomorphic objects, for example one may freely cut, paste and interpolate various holomorphic objects), and then check whether $F$ has Thurston obstructions (this is not always easy). If not then Thurston's theorem ensures the existence of a rational map with the same combinatorial properties.

In practice, one sometimes needs a slightly generalized version of Thurston's theorem, namely one with a larger marked set than the mere postcritical set. We will call it 'marked Thurston's theorem'. The main purpose of writing up this chapter is to provide a self-contained proof of this theorem. As one can see below, the proof follows essentially the same line as that presented by Douady and Hubbard ([DH1]), except some refinements in the estimates. For instance to get a strong contraction of the pullback operator on the appropriate Teichmüller space, we had to raise the operator to a large power (instead of just to its second power).

Just to illustrate the power of Thurston's characterization theorem we will mention some of its applications. There are many such applications. These include Rees' descriptions of parameter spaces [Re2], Kiwi's characterization of polynomial laminations (using previous work of Bielefield-Fisher-Hubbard $[\mathrm{BFH}]$ and Poirier [Po]), Rees, Shishikura and Tan's studies on matings of polynomials ([Re1, ST, Ta1, Ta2]), Pilgrim and Tan's cut-and-paste surgery along $\operatorname{arcs}([\mathrm{PT}])$, and Timorin's topological regluing of rational maps ([Ti]), among many others. Furthermore, one of the two main outstanding questions in the field, namely, the density of hyperbolicity in the quadratic polynomial family, 
can be reduced to the assertion that every (infinitely renormalizable) quadratic polynomial $p$ is a limit of certain postcritically finite ones $p_{n}$ obtained via Thurston's theorem and McMullen's quotienting process ([McM]). The detailed knowledge of the combinatorics of the parameter space of quadratic polynomials (which follows from a special case of Thurston's theorem) was used by Sørensen ([So]) to construct highly non-hyperbolic quadratic polynomials with non-locally connected Julia sets, and this in turn was used by Henriksen ([Hen]) to show that McMullen's combinatorial rigidity property fails for cubic polynomials.

We will give a more complete, but by no means exhaustive, list of applications and related results. We mention in particular an interesting result of $\mathrm{L}$. Geyer beyond the field of complex dynamics. Khavinson and Świątek $([\mathrm{KS}])$ proved that harmonic polynomials $z-\overline{p(z)}$, where $p$ is a holomorphic polynomial of degree $n>1$, have at most $3 n-2$ roots, and the bound is sharp for $n=2,3$. Bshouty and Lyzzaik ([BL]) extended the sharpness of the bound to the cases $n=4,5,6$ and 8, using purely algebraic methods. Finally L. Geyer ([Ge]) settled the sharpness for all $n$ at once, by constructing 'à la Thurston' a polynomial $p$ of degree $n$ with real coefficients and with mutually distinct critical points $z_{1}, z_{2}, \ldots, z_{n-1}$ such that $\overline{p\left(z_{j}\right)}=z_{j}$.

We will also present the notion of deformation space of a rational map introduced by Adam Epstein in his PhD thesis (in fact, the construction applies to finite type transcendental maps on compact Riemann surfaces which was his original motivation). Those are smooth sub-manifolds of appropriate Teichmüller spaces of spheres with marked points. In the dynamical setting, the relation between Epstein's deformation spaces and spaces of rational maps is somewhat comparable to the relation between Teichmüller spaces and moduli spaces in the classical theory of Riemann surfaces. Interesting transversality properties are more easily expressed and proved in those deformation spaces, and we believe they will attract an increasing amount of interest in the coming years.

\section{Teichmüller spaces for rational maps}

In this section we will recall the classical theory of the Teichmüller space of a marked sphere, define the Teichmüller space associated to a rational map, the Thurston's pullback map and the Epstein's deformation space.

\subsection{The Teichmüller space of a marked sphere}

Let $S^{2}$ be an oriented surface homeomorphic to $\widehat{\mathbb{C}}$. All homeomorphisms $S^{2} \rightarrow$ $\widehat{\mathbb{C}}$ we will consider are orientation preserving. 
Let $Z \subset S^{2}$ be finite with $\# Z \geq 4$. Then,

- $\mathbf{M}_{Z}$ is the space of equivalence classes $[i]$, with $i: Z \hookrightarrow \widehat{\mathbb{C}}$ an injection and $i_{1} \sim i_{2}$ if there is a Möbius transformation $M$ such that $M \circ i_{1}=i_{2}$.

- $\mathcal{T}_{Z}=\operatorname{Teich}\left(S^{2}, Z\right)$, the Teichmüller space of the marked sphere $\left(S^{2}, Z\right)$, is defined to be the space of equivalence classes of homeomorphisms $\phi: S^{2} \rightarrow \widehat{\mathbb{C}}$ with $\phi \sim \varphi$, if there is a Möbius transformation $M$ such that $\left.M \circ \varphi\right|_{Z}=\left.\phi\right|_{Z}$ and $M \circ \varphi=\phi \circ h$, with $h$ a homeomorphism isotopic to the identity rel $Z$. Here is the diagram:

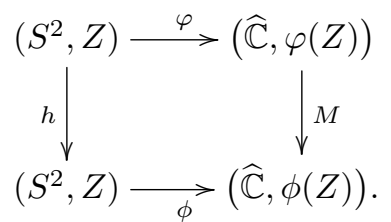

- For a finite set $X \subset \widehat{\mathbb{C}}$ containing at least three points, we denote by $\mathcal{Q}(X)$ the space of integrable quadratic differentials on $\widehat{\mathbb{C}}$ which are holomorphic outside $X$. Equivalently, $\mathcal{Q}(X)$ is the space of meromorphic quadratic differentials on $\widehat{\mathbb{C}}$, holomorphic outside $X$ with at worst simple poles in $X$. By the Riemann-Roch theorem, the number of poles minus the number of zeros of a meromorphic quadratic differential on $\widehat{\mathbb{C}}$ is equal to 4 , taking into account multiplicities. It follows that $\mathcal{Q}(X)$ is a $\mathbb{C}$-linear space of dimension

$$
\operatorname{dim} \mathcal{Q}(X)=\# X-3 .
$$

- The space $\mathcal{Q}(X)$ is equipped with the norm

$$
\|q\|=\int_{\widehat{\mathbb{C}}}|q|=\int_{\mathbb{C}}|q(x+i y)| \mathrm{d} x \mathrm{~d} y .
$$

If $\psi: S^{2} \rightarrow \widehat{\mathbb{C}}$ represents a point $\tau \in \mathcal{T}_{Z}$, the cotangent space to $\mathcal{T}_{Z}$ at $\tau$ may be canonically identified to $\mathcal{Q}(\psi(Z))$.

- We equip $T_{\tau} \mathcal{T}_{Z}$ with the dual norm

$$
\forall \nu \in T_{\tau} \mathcal{T}_{Z}, \quad\|\nu\|=\sup _{\substack{q \in \mathcal{Q}(\psi(Z)) \\\|q\| \leq 1}}|\langle q, \nu\rangle| .
$$

- The induced Teichmüller metric on $\mathcal{T}_{Z}$ is given by

$$
d_{\mathcal{T}_{Z}}\left(\left[\phi_{1}\right],\left[\phi_{2}\right]\right)=\inf \frac{1}{2} \log K(h)
$$

where the infimum is taken over all the quasiconformal homeomorphisms $h: \widehat{\mathbb{C}} \rightarrow \widehat{\mathbb{C}}$ such that $\phi_{1}^{-1} \circ h \circ \phi_{2}$ is homotopic to the identity rel $Z$ and 
where $K(h)$ is the quasiconformal distortion

$$
K(h)=\frac{1+\|\bar{\partial} h / \partial h\|_{\infty}}{1-\|\bar{\partial} h / \partial h\|_{\infty}} .
$$

\subsection{The Teichmüller space of a rational map}

Let $f: \widehat{\mathbb{C}} \rightarrow \widehat{\mathbb{C}}$ be a rational map with $\operatorname{deg}(f) \geq 2$.

The grand orbit of a point $z$ is defined to be

$$
\left\{z^{\prime} \in \widehat{\mathbb{C}} \mid \exists n, m \in \mathbb{N} \text { such that } f^{n}\left(z^{\prime}\right)=f^{m}(z)\right\} .
$$

The extended Julia set, denoted by $\widehat{\mathcal{J}}_{f}$, is the closure of the grand orbits of all periodic points and all critical points. We always have

$$
\mathcal{P}_{f} \cup \mathcal{J}_{f} \subseteq \widehat{\mathcal{J}}_{f} .
$$

- $\mathbf{M}(f)$, the moduli space of $f$, denotes the space of conformal equivalence classes of rational maps quasiconformally conjugate to $f$, that is

$$
\mathbf{M}(f)=\left\{\begin{array}{l|l}
g & \begin{array}{l}
\text { there is a quasiconformal map } h \\
\text { such that } h \circ f=g \circ h
\end{array}
\end{array}\right\} / \sim
$$

where $g \sim G$ if there is a Möbius transformation $H$ such that $g \circ H=$ $H \circ G$.

- $\mathrm{QC}(f)$ is the group of quasiconformal automorphisms of $\widehat{\mathbb{C}}$ which commute with $f$.

- $\mathrm{QC}_{0}(f) \subset \mathrm{QC}(f)$ is the normal subgroup consisting of those quasiconformal automorphisms which are isotopic to the identity in an appropriate sense: there is a family $\left(h_{t}\right), t \in[0,1]$, with $h_{0}=\mathrm{id}, h_{1}=h$ such that each $h_{t}$ is quasiconformal, $h_{t} \circ f=f \circ h_{t}$, and $(t, z) \mapsto\left(t, h_{t}(z)\right)$ is a homeomorphism from $[0,1] \times \widehat{\mathbb{C}}$ onto itself. Note that $h_{t}$ must be the identity on the set of periodic points as well as on the postcritical set for all $t \in[0,1]$. Consequently $h_{t}$ is the identity on $\widehat{\mathcal{J}}_{f}$.

- $\operatorname{Mod}(f)=\mathrm{QC}(f) / \mathrm{QC}_{0}(f)$, the modular group of $f$, denotes the group of isotopy classes of quasiconformal automorphisms of $f$ up to isotopy, that is, the group of equivalence classes $[\phi]$, such that $\phi: \widehat{\mathbb{C}} \rightarrow \widehat{\mathbb{C}}$ is a quasiconformal homeomorphism, $\phi \circ f=f \circ \phi$, and $\phi \sim \varphi$ if $\varphi=\phi \circ h$ with $h \in \mathrm{QC}_{0}(f)$. This group contains as a subgroup the set of Möbius transformations commuting with $f$, denoted by $\operatorname{Aut}(f)$.

- $\mathcal{T}(f)$, the Teichmüller space of $f$ is the set of equivalence classes of pairs $(g, \psi)$ such that $g$ is a rational map, $\psi$ is a quasiconformal conjugacy between $f$ and $g$ (i.e. $\psi \circ f=g \circ \psi)$, and $\left(g_{1}, \psi_{1}\right) \sim\left(g_{2}, \psi_{2}\right)$ if there is a 
Möbius transformation $M$ such that $g_{1}=M \circ g_{2} \circ M^{-1}$ and $M \circ \psi_{2}=\psi_{1} \circ h$ with $h \in \mathrm{QC}_{0}(f)$ :

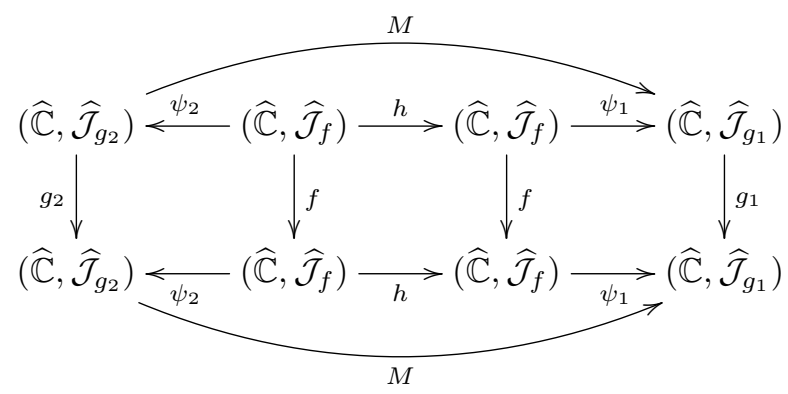

For example when $M \in \operatorname{Aut}(f)$, then $(f, M) \sim(f$,id). Note that

$$
\mathbf{M}(f)=\mathcal{T}(f) / \operatorname{Mod}(f) .
$$

Let Rat $_{d}$ denote the space of all rational maps $f: \widehat{\mathbb{C}} \rightarrow \widehat{\mathbb{C}}$ of degree $d$. This space can be realized as the complement of a hyper-surface in the projective space $\mathbb{P}^{2 d+1}(\mathbb{C})$ by considering $f(z)=p(z) / q(z)$ where $p$ and $q$ are relatively prime polynomials in $z$ with $d=\max \{\operatorname{deg} p, \operatorname{deg} q\}$. The group of Möbius transformations Aut $(\widehat{\mathbb{C}})$ acts on $\operatorname{Rat}_{d}$ by conjugacy: if $\phi \in \operatorname{Aut}(\widehat{\mathbb{C}})$ and $f \in$ $\operatorname{Rat}_{d}$, then $\phi \cdot f=\phi^{-1} \circ f \circ \phi \in \operatorname{Rat}_{d}$.

Theorem 1.1 (McMullen and Sullivan, [MS]). The group $\operatorname{Mod}(f)$ acts properly discontinuously by holomorphic automorphisms on $\mathcal{T}(f)$. There is a natural holomorphic injection of complex orbifolds $\mathbf{M}(f) \rightarrow \operatorname{Rat}_{d} / \operatorname{Aut}(\widehat{\mathbb{C}})$ parameterizing the rational maps $g$ quasiconformally conjugate to $f$.

Each connected component of the Fatou set $\mathcal{F}$ of a rational map $f$ of degree $d \geq 2$ properly maps to a connected component of $\mathcal{F}$. Such a Fatou component $U$ is periodic if there is a $p \geq 1$ such that $f^{p}(U)=U$ and is preperiodic if $f^{k}(U)$ is periodic for some $k \geq 0$. If $U$ is not preperiodic, then it is called a wandering Fatou component.

Sullivan, using the Measurable Riemann Mapping Theorem in Teichmüller theory, proved that if $f$ had a wandering Fatou component, then the Teichmüller space $\mathcal{T}(f)$ would be infinite dimensional, contradicting the previous theorem since $\operatorname{Rat}_{d} / \operatorname{Aut}(\widehat{\mathbb{C}})$ has dimension $2 d-2$. Thus,

Theorem 1.2 (Sullivan, $[\mathrm{Su}]$ ). Every Fatou component of a rational map is preperiodic.

Since Sullivan, the Measurable Riemann Mapping Theorem has been applied in almost every domain of holomorphic dynamics. We recommend the 
monograph of Branner-Fagella, [BF], for a detailed account of relative results and references.

The following classification of periodic Fatou components goes back to Fatou and is rather elementary. Assume $U$ is a periodic Fatou component of period $p$. Then $U$ is either

- a superattracting basin: there is a point $z_{0}$ in $U$, fixed by $f^{p}$, with $\left(f^{p}\right)^{\prime}\left(z_{0}\right)=0$, attracting all points of $U$ under iteration of $f^{p}$;

- an attracting basin: there is a point $z_{0}$ in $U$, fixed by $f^{p}$, with $0<$ $\left|\left(f^{p}\right)^{\prime}\left(z_{0}\right)\right|<1$, attracting all points of $U$ under iteration of $f^{p}$;

- a parabolic basin: there is a point $z_{0}$ in $\partial U$ with $\left(f^{p}\right)^{\prime}\left(z_{0}\right)=1$, attracting all points of $U$;

- a Siegel disk: $U$ is conformally isomorphic to the unit disk, and $\left.f^{p}\right|_{U}$ is conformally conjugate to an irrational rotation;

- a Herman ring: $U$ is conformally isomorphic to an annulus $\{r<|z|<R\}$ with $0<r<R<\infty$, and $\left.f^{p}\right|_{U}$ is conformally conjugate to an irrational rotation.

If $U$ is an attracting basin, then $f$ acts properly discontinuously on $U \backslash \widehat{\mathcal{J}}_{f}$ and the quotient $\left(U \backslash \widehat{\mathcal{J}}_{f}\right) / f$ is isomorphic to a punctured torus. If $U$ is a parabolic basin, then $f$ acts properly discontinuously on $U \backslash \widehat{\mathcal{J}}_{f}$ and the quotient $\left(U \backslash \widehat{\mathcal{J}}_{f}\right) / f$ is isomorphic to a punctured sphere.

Theorem 1.3 (McMullen and Sullivan, $[\mathrm{MS}]$ ). The space $\mathcal{T}(f)$ is canonically isomorphic to a connected finite-dimensional complex manifold, which is the product of a polydisk and the traditional Teichmüller spaces associated to punctured tori and punctured spheres.

In particular, the obstruction to deforming a quasiconformal conjugacy between two rational maps to a conformal conjugacy is measured by finitely many complex moduli.

\subsection{Thurston's pullback map}

Let $F: S^{2} \rightarrow S^{2}$ be an orientation preserving branched covering of degree $d \geq 2$. The set $\mathcal{C}_{F}$ of critical points, the set $\mathcal{V}_{F}$ of critical values and the postcritical set $\mathcal{P}_{F}$ are defined in the same way as for a rational map.

Assume $Y \subset S^{2}$ is a finite set containing at least three points with $\mathcal{V}_{F} \subseteq Y$. Then there is a Thurston's pullback map $\varsigma_{F}: \mathcal{T}_{Y} \rightarrow \mathcal{T}_{F^{-1}(Y)}$ which may be defined as follows. Let $\tau \in \mathcal{T}_{Y}$ be represented by a homeomorphism $\phi$ : $S^{2} \rightarrow \widehat{\mathbb{C}}$. This homeomorphism $\phi$ defines a complex structure $\mathfrak{c}$ on $S^{2}$ which can be pulled-back via $F: S^{2} \rightarrow S^{2}$ to a complex structure $F^{*} \mathfrak{c}$ on $S^{2}$ (one 
has to use the removable singularity theorem to define the complex structure near the critical points of $F$ ). The Uniformization Theorem guarantees the existence of an isomorphism $\psi:\left(S^{2}, F^{*} \mathfrak{c}\right) \rightarrow \widehat{\mathbb{C}}$. Then, $\varsigma_{F}$ is defined by

$$
\mathcal{T}_{Y} \ni[\phi] \stackrel{\varsigma_{F}}{\longrightarrow}[\psi] \in \mathcal{T}_{F^{-1}(Y)} .
$$

It is not obvious that this definition is independent on the choice of $\phi$ and $\psi$. We will show this now.

First, note that $\phi \circ F \circ \psi^{-1}$ is analytic (thus a rational map):

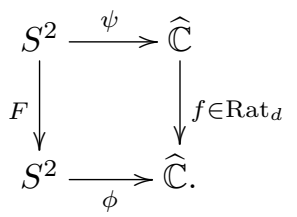

Assume $\tau \in \mathcal{T}_{Y}$ is represented by the homeomorphisms $\phi_{0}: S^{2} \rightarrow \widehat{\mathbb{C}}$ and $\phi_{1}: S^{2} \rightarrow \widehat{\mathbb{C}}$. Let $M: \widehat{\mathbb{C}} \rightarrow \widehat{\mathbb{C}}$ be a Möbius transformation and let $h: S^{2} \rightarrow S^{2}$ be a homeomorphism isotopic to the identity rel $Y$, such that $M=\phi_{0} \circ h \circ \phi_{1}^{-1}$. Let $\psi_{0}: S^{2} \rightarrow \widehat{\mathbb{C}}$ and $f_{0}: \widehat{\mathbb{C}} \rightarrow \widehat{\mathbb{C}}$ (resp. $\psi_{1}: S^{2} \rightarrow \widehat{\mathbb{C}}$ and $f_{1}: \widehat{\mathbb{C}} \rightarrow \widehat{\mathbb{C}}$ ) satisfy diagram (1.2). Since $Y \supseteq \mathcal{V}_{F}$, there is a lift $k: S^{2} \rightarrow S^{2}$ which is a homeomorphism isotopic to the identity rel $F^{-1}(Y)$ such that $h \circ F=F \circ k$. We therefore have a commutative diagram:

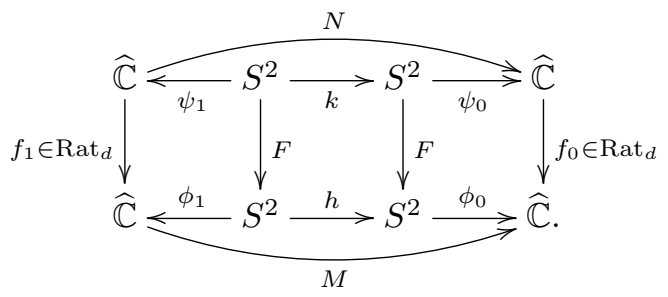

Since $M, f_{0}$ and $f_{1}$ are analytic, the homeomorphism $N=\psi_{0} \circ k \circ \psi_{1}^{-1}$ is analytic, thus a Möbius transformation. As a consequence, $\psi_{0}$ and $\psi_{1}$ represent the same point in $\mathcal{T}_{F^{-1}(Y)}$.

Proposition 1.4. The map $\varsigma_{F}: \mathcal{T}_{Y} \rightarrow \mathcal{T}_{F^{-1}(Y)}$ is analytic.

Proof. Let $\psi: S^{2} \rightarrow \widehat{\mathbb{C}}$ and $\phi: S^{2} \rightarrow \widehat{\mathbb{C}}$ be such that $f=\phi \circ F \circ \psi^{-1} \in$ Rat $_{d}$. The Teichmüller spaces $\mathcal{T}_{Y}$ and $\mathcal{T}_{F^{-1}(Y)}$ are canonically identified to quotients of the unit ball of the space of Beltrami differentials on $\widehat{\mathbb{C}}$ and the map $\varsigma_{F}$ : $\mathcal{T}_{Y} \rightarrow \mathcal{T}_{F^{-1}(Y)}$ is induced by the $\mathbb{C}$-linear (thus analytic) map $\mu \mapsto f^{*} \mu$.

Assume now $X \subseteq F^{-1}(Y)$ contains at least three points. Then, there is an analytic submersion $\varpi: \mathcal{T}_{F^{-1}(Y)} \rightarrow \mathcal{T}_{X}$ which consists in forgetting points in 
$F^{-1}(Y) \backslash X$. We shall use the notation $\sigma_{F}$ for the Thurston's pullback map

$$
\sigma_{F}=\varpi \circ \varsigma_{F}: \mathcal{T}_{Y} \rightarrow \mathcal{T}_{X} .
$$

As a composition of analytic maps, this map is itself analytic. We will be particularly interested in the case that $F$ is postcritically finite (i.e., $\mathcal{P}_{F}$ is finite) and $X=Y=\mathcal{P}_{F}$.

Now, if $f: \widehat{\mathbb{C}} \rightarrow \widehat{\mathbb{C}}$ is a rational map and $q$ is a meromorphic quadratic differential on $\widehat{\mathbb{C}}$, the pullback $f^{*} q$ and the push forward $f_{*} q$ may be defined in coordinates as follows:

- if $q=b(y) \mathrm{d} y^{2}$, then $f^{*} q=a(x) \mathrm{d} x^{2}$ with $a(x)=b(f(x)) \cdot\left(f^{\prime}(x)\right)^{2}$.

- if $q=b(y) \mathrm{d} y^{2}$, then $f_{*} q=c(z) \mathrm{d} z^{2}$ with $c(f(y))=\sum_{y \in f^{-1}(z)} \frac{b(y)}{\left(f^{\prime}(y)\right)^{2}}$.

It follows that

$$
\begin{aligned}
& f\left(\left\{\operatorname{poles}\left(f^{*} q\right)\right\}\right) \subseteq\{\operatorname{poles}(q)\} \quad \text { and } \\
& f^{-1}(\{\operatorname{poles}(q)\}) \subseteq\left\{\operatorname{poles}\left(f^{*} q\right) \cup \mathcal{C}_{f}\right\} ;
\end{aligned}
$$

on the other hand,

$$
\left\{\operatorname{poles}\left(f_{*} q\right)\right\} \subseteq f\left(\mathcal{C}_{f}\right) \cup f(\{\operatorname{poles}(q)\}) .
$$

Let $\tau \in \mathcal{T}_{Y}$ be represented by $\phi: S^{2} \rightarrow \widehat{\mathbb{C}}$. Let $\psi: S^{2} \rightarrow \widehat{\mathbb{C}}$ represent $\sigma_{F}(\tau) \in \mathcal{T}_{X}$ with $f=\phi \circ F \circ \psi^{-1} \in \operatorname{Rat}_{\mathrm{d}}$. Then, the cotangent space to $\mathcal{T}_{Y}$ at $\tau$ is canonically identified to $\mathcal{Q}(\phi(Y))$ and the cotangent space to $\mathcal{T}_{X}$ at $\sigma_{F}(\tau)$ is canonically identified to $\mathcal{Q}(\psi(X))$. By means of those identifications, the adjoint map of the derivative $D_{\tau} \sigma_{F}: T_{\tau} \mathcal{T}_{Y} \rightarrow T_{\sigma_{F}(\tau)} \mathcal{T}_{X}$ is the push forward operator $f_{*}: \mathcal{Q}(\psi(X)) \rightarrow \mathcal{Q}(\phi(Y))$.

\subsection{Epstein's deformation space}

In his Ph.D. thesis, generalizing a construction due to Thurston which will be described below, Adam Epstein introduced the following deformation space.

Definition. Let $F: S^{2} \rightarrow S^{2}$ be an orientation preserving branched covering of degree $d \geq 2$. Let $X$ and $Y$ be finite subsets of $\widehat{\mathbb{C}}$ containing at least three points such that $X \subseteq Y \cap F^{-1}(Y)$ and $\mathcal{V}_{F} \subseteq Y$. Define

$$
\operatorname{Def}_{X}^{Y}(F)=\left\{\tau \in \mathcal{T}_{Y} \mid \pi(\tau)=\sigma_{F}(\tau)\right\},
$$

where $\pi: \mathcal{T}_{Y} \rightarrow \mathcal{T}_{X}$ is the submersion which consists in forgetting points in $Y \backslash X$ and $\sigma_{F}: \mathcal{T}_{Y} \rightarrow \mathcal{T}_{X}$ is the Thurston's pullback map induced by $F$.

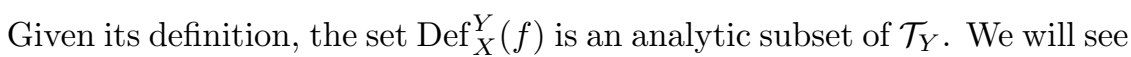
that in most cases, it is either empty or a smooth submanifold of $\mathcal{T}_{Y}$ (Theorem 
1.5 below). We may first briefly discuss why this space is interesting from a dynamical point of view.

Note that if $\phi: S^{2} \rightarrow \widehat{\mathbb{C}}$ represents a point $\tau \in \operatorname{Def}_{X}^{Y}(F)$, then, there is a unique $\psi: S^{2} \rightarrow \widehat{\mathbb{C}}$ representing $\pi(\tau)=\sigma_{F}(\tau)$ and coinciding with $\phi$ on $X$. In that case, the map $f=\phi \circ F \circ \psi^{-1}$ is a rational map of degree $d$ and we have the following commutative diagram:

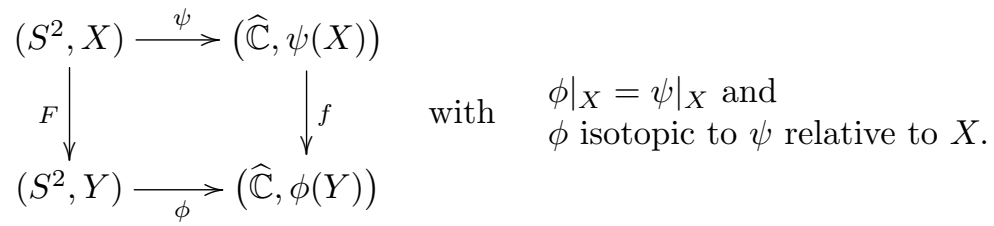

Any point of $\operatorname{Def}_{X}^{Y}(F)$ is represented by a triple $(\phi, \psi, f)$ as in this diagram. If $\left(\phi_{1}, \psi_{1}, f_{1}\right)$ and $\left(\phi_{2}, \psi_{2}, f_{2}\right)$ are two triples representing the same point $\tau \in$ $\operatorname{Def}_{X}^{Y}(F)$, then the rational maps $f_{1}$ and $f_{2}$ are Möbius conjugate by the Möbius transformation sending $\psi_{1}(X)$ to $\psi_{2}(X)$. In particular, there is a natural map

$$
\Phi: \operatorname{Def}_{X}^{Y}(F) \rightarrow \operatorname{Rat}_{d} / \operatorname{Aut}(\widehat{\mathbb{C}}) .
$$

In addition, for $x \in X$ we have

$$
f(\phi(x))=\phi(F(x)) .
$$

In particular, $\phi$ sends cycles of $F$ contained in $X$ to cycles of $f$.

If $F$ is postcritically finite, there exists a smallest function

$$
\nu_{F}: S^{2} \rightarrow \mathbb{N} \cup\{\infty\}
$$

such that $\nu(x)=1$ if $x \notin \mathcal{P}_{F}$ and $\nu(x)$ is a multiple of $\nu(y) \cdot \operatorname{deg}_{y} F$ for each $y \in F^{-1}(x)$. The function $\nu_{F}$ is called the orbifold signature of $F$.

We say that an orientation-preserving branched covering $F$ is a $\mathbf{( 2 , 2 , 2 , 2 ) -}$ map if $F$ is postcritically finite and its orbifold signature takes the value 2 exactly at 4 points and the value 1 otherwise. This happens exactly when $\# \mathcal{P}_{F}=4, \mathcal{C}_{F} \cap \mathcal{P}_{F}=\emptyset$ and all critical points of $F$ are simple.

Theorem 1.5 (Epstein,[E2]). If $F$ is not a $(2,2,2,2)$-map or if $X$ does not contain the entire postcritical set of $F$, then the deformation space $\operatorname{Def}_{X}^{Y}(F)$ is either empty or a smooth manifold of $\mathcal{T}_{Y}$ of dimension $\#(Y-X)$.

Proof. Let $\tau$ be a point of $\operatorname{Def}_{X}^{Y}(F)$ represented by a triple $(\phi, \psi, f)$. By the Implicit Function Theorem, it is enough to show that the linear map

$$
D_{\tau} \pi-D_{\tau} \sigma_{F}: T_{\tau} \mathcal{T}_{Y} \rightarrow T_{\pi(\tau)} \mathcal{T}_{X}
$$

is surjective. The cotangent space to $\mathcal{T}_{Y}$ at $\tau$ is canonically identified to $\mathcal{Q}(\phi(Y))$ and the cotangent space to $\mathcal{T}_{X}$ at $\pi(\tau)=\sigma_{F}(\tau)$ is canonically 
identified to $\mathcal{Q}(\phi(X))$. The adjoint map of $D_{\tau} \pi-D_{\tau} \sigma_{F}$ is the linear map $\nabla_{f}=\mathrm{id}-f_{*}: \mathcal{Q}(\phi(X)) \rightarrow \mathcal{Q}(\phi(Y))$. It is enough to prove that this linear map is injective. If there were a $q \in \mathcal{Q}(\phi(X))$ such that $q=f_{*} q$, according to Lemma 1.6 below, $f$ would be a $(2,2,2,2)$-map and the set of poles of $q$ would be $\mathcal{P}_{f}$. As a consequence, we would have $\mathcal{P}_{f} \subseteq \phi(X)$. The restriction of $F$ to $X$ is conjugate to the restriction of $f$ to $\phi(X)$. Thus, $F$ would be a $(2,2,2,2)$-map with $\mathcal{P}_{F} \subseteq X$, contradicting our assumptions.

Lemma 1.6 (Thurston's contraction principle, [Th1, DH1]). Let $f: \widehat{\mathbb{C}} \rightarrow \widehat{\mathbb{C}}$ be a rational map of degree $d \geq 2$. Then for any integrable meromorphic quadratic differential $q$ on $\widehat{\mathbb{C}}$, we have $\left\|f_{*} q\right\| \leq\|q\|$, with equality if and only if $f^{*} f_{*} q=d \cdot q$. Furthermore, if $q=f_{*} q$ for some $q \neq 0$ then $f$ is a $(2,2,2,2)$ map and the set of poles of $q$ is $\mathcal{P}_{f}$.

Proof. The inequality $\left\|f_{*} q\right\| \leq\|q\|$ follows easily from the triangle inequality: if $U \subset \widehat{\mathbb{C}} \backslash \mathcal{V}_{f}$ is a simply connected open set of full measure and if $\left\{g_{i}\right\}_{i \in\{1, \ldots, d\}}$ are the inverse branches of $f$ on $U$, then

$$
\int_{\widehat{\mathbb{C}}}\left|f_{*} q\right|=\int_{U}\left|f_{*} q\right|=\int_{U}\left|\sum_{g_{i}} g_{i}^{*} q\right| \leq \int_{U} \sum_{g_{i}}\left|g_{i}^{*} q\right|=\int_{f^{-1}(U)}|q| \leq \int_{\widehat{\mathbb{C}}}|q| .
$$

The case of equality follows easily.

As a consequence, if $q=f_{*} q$, we have $f^{*} q=d \cdot q$. In particular, the set $Z$ of poles of $q$ satisfies $f(Z) \subseteq Z$ and $f^{-1}(Z) \subseteq Z \cup \mathcal{C}_{f}$. Thus,

$$
\# Z+(2 d-2) \geq \# Z+\# \mathcal{C}_{f} \geq \# f^{-1}(Z) \geq d \cdot \# Z-(2 d-2) .
$$

This implies $4(d-1) \geq \# Z(d-1)$. As $d>1$, we have $\# Z \leq 4$.

Assume $q \neq 0$. Then, $q$ has at least 4 poles, thus $\# Z=4$ and all inequalities in (1.6) become equalities. The leftmost equality in (1.6) implies $Z \cap \mathcal{C}_{f}=\emptyset$ and $\# \mathcal{C}_{f}=2 d-2$, which means that all critical points of $f$ are simple. The middle equality means that $f^{-1}(Z)=Z \sqcup \mathcal{C}_{f}$ so $\mathcal{V}_{f} \subseteq Z$. But $f(Z) \subseteq Z$ (if $q$ has a pole at $z$, then $f_{*} q$ has a pole at $\left.f(z)\right)$. So $\mathcal{P}_{f}=\bigcup_{n>0} f^{n}\left(\mathcal{V}_{f}\right) \subseteq Z$. It remains to show $Z \subseteq \mathcal{P}_{f}$. Note that $f^{-1}\left(Z \backslash \mathcal{P}_{f}\right)$ is contained in $Z \cup \mathcal{C}_{f}$ and is disjoint from $\mathcal{C}_{f} \cup \mathcal{P}_{f}$. So $f^{-1}\left(Z \backslash \mathcal{P}_{f}\right) \subseteq Z \backslash \mathcal{P}_{f}$ and hence $f^{-n}\left(Z \backslash \mathcal{P}_{f}\right) \subseteq Z \backslash \mathcal{P}_{f}$ for any $n$. But $f^{-1}(z)$ consists of $d$ distinct points for any $z$ which is not a critical value. This proves that $Z \backslash \mathcal{P}_{f}$, as a set with at most 4 points, must be empty. Therefore $f$ is a $(2,2,2,2)$-map and the set of poles of $q$ is $\mathcal{P}_{f}$.

Corollary 1.7. Let $f$ be a rational map of degree $d>2$ that is not a (2,2,2,2)map. Then the operator $\nabla_{f}=\mathrm{id}-f_{*}$ is injective on the space of integrable meromorphic quadratic differentials on $\widehat{\mathbb{C}}$.

Characterizing the cases for which the deformation space $\operatorname{Def}_{X}^{Y}(f)$ is not empty is not an easy task. Thurston's theorem below gives precise conditions 
under which this space is not empty (actually is a single point) when $F$ is postcritically finite and $X=Y=Z$ is a finite forward invariant set containing $\mathcal{P}_{F}$.

\section{Thurston's theorem with marked points}

Let us define an equivalence relation on the set of pairs $(F, Z)$ such that $F$ : $S^{2} \rightarrow S^{2}$ is an orientation-preserving branched covering of a topological sphere $S^{2}$ of degree $\operatorname{deg}(F) \geq 2$ and $Z \subset S^{2}$ is a finite set satisfying $\mathcal{P}_{F} \subseteq Z$ and $F(Z) \subseteq Z$.

An equivalence $(\phi, \psi)$ between two pairs $\left(F_{0}, Z_{0}\right)$ and $\left(F_{1}, Z_{1}\right)$ is a pair of homeomorphisms $\phi, \psi: S^{2} \rightarrow S^{2}$ such that $\phi\left(Z_{0}\right)=\psi\left(Z_{0}\right)=Z_{1},\left.\phi\right|_{Z_{0}}=\left.\psi\right|_{Z_{0}}$, the two maps $\phi$ and $\psi$ are isotopic rel $Z_{0}$ and $F_{1} \circ \psi=\phi \circ F_{0}$. In this situation, we say that $\left(F_{0}, Z_{0}\right)$ is combinatorially equivalent to $\left(F_{1}, Z_{1}\right)$.

In the case that $Z=\mathcal{P}_{F}$ and $\# Z<\infty$, Thurston's characterization theorem ([Th1, DH1]) provides a necessary and sufficient condition for $(F, Z)$ to be combinatorially equivalent to $(f, X)$ with $f: \widehat{\mathbb{C}} \rightarrow \widehat{\mathbb{C}}$ a rational map (we say that $(f, X)$ is a rational representative). We will now present the condition.

\subsection{Thurston obstructions}

A Jordan curve $\gamma$ disjoint from $Z$ is said null-homotopic (resp. peripheral) rel $Z$ if one of its complementary component contains zero (resp. one) point of $Z$. A Jordan curve that is disjoint from $Z$, such that each of its two complementary components contains at least two points of $Z$, is said non-peripheral rel $Z$.

We say that $\Gamma=\left\{\gamma_{1}, \cdots, \gamma_{k}\right\}$ is a multicurve of $(F, Z)$, if each $\gamma_{i}$ is a Jordan curve disjoint from $Z$ and is non-peripheral rel $Z$, and the $\gamma_{j}$ 's are mutually disjoint and mutually non-homotopic rel $Z$.

We say that $\Gamma$ is $(F, Z)$-stable if every curve of $F^{-1}(\Gamma)$ is either homotopic rel $Z$ to a curve of $\Gamma$ or null-homotopic or peripheral rel $Z$. This implies that for any $m>0$, every curve of $F^{-m}(\Gamma)$ is either homotopic rel $Z$ to a curve of $\Gamma$ or null-homotopic or peripheral $\operatorname{rel} Z$.

Each such $\Gamma$ induces an $(F, Z)$-transition matrix $F_{\Gamma}$ together with its leading eigenvalue $\lambda_{\Gamma}$ as follows: Let $\left(\gamma_{i, j, \delta}\right)_{\delta}$ denote the components of $F^{-1}\left(\gamma_{j}\right)$ homotopic to $\gamma_{i}$ rel $Z$ (there might be no such components). Then $F: \gamma_{i, j, \delta} \rightarrow \gamma_{j}$ is a topological covering of a certain degree $d_{i, j, \delta}$. The transition matrix is defined to be $F_{\Gamma}=\left(\sum_{\delta} 1 / d_{i, j, \delta}\right)$. This is a non-negative matrix. By PerronFrobenius Theorem there is a non-negative eigenvalue $\lambda_{\Gamma}$ that coincides with the spectral radius of $F_{\Gamma}$. 
We say that an $(F, Z)$-stable multicurve $\Gamma$ is a Thurston obstruction for $(F, Z)$ if $\lambda_{\Gamma} \geq 1$. In the particular case $Z=\mathcal{P}_{f}$, we simply say that $\Gamma$ is a Thurston obstruction for $F$.

\subsection{Main results}

Theorem 2.1 (Marked Thurston's theorem). Let $F: S^{2} \rightarrow S^{2}$ be a postcritically finite branched covering which is not a $(2,2,2,2)$-map. Let $Z \subset S^{2}$ be finite with $\mathcal{P}_{F} \subseteq Z$ and $F(Z) \subseteq Z$. If $(F, Z)$ has no Thurston obstructions, then the combinatorial equivalence class of $(F, Z)$ contains a rational representative which is unique up to Möbius conjugacy. More precisely, if $(\phi, \psi)$ is an equivalence between two rational representatives $\left(f_{1}, X_{1}\right)$ and $\left(f_{2}, X_{2}\right)$, then there is a (unique) Möbius transformation $M$ which is isotopic to both $\phi$ and $\psi$ rel $X_{1}$ and satisfies $M \circ f_{1}=f_{2} \circ M$.

Remark. Our statement is slightly more general than Thurston's original theorem (see [Th1, DH1]), where $Z=\mathcal{P}_{F}$. We actually prove more.

Theorem 2.2. Let $F: S^{2} \rightarrow S^{2}$ be a branched covering and $Z \subset S^{2}$ be a finite set containing at least three points $x_{0}, x_{1}, x_{2}$ with $\mathcal{P}_{F} \subseteq Z$ and $F(Z) \subseteq Z$. Let $\phi_{0}: S^{2} \rightarrow \widehat{\mathbb{C}}$ be any given orientation preserving homeomorphism. Define $\left(\phi_{n}, f_{n}\right)$ recursively so that $\phi_{n}: S^{2} \rightarrow \widehat{\mathbb{C}}$ is a homeomorphism agreeing with $\phi_{0}$ on $\left\{x_{0}, x_{1}, x_{2}\right\}$ and so that the map $f_{n}=\phi_{n-1} \circ F \circ \phi_{n}^{-1}$ is a rational map. If $F$ is not a $(2,2,2,2)$-map and $(F, Z)$ has no Thurston obstructions, then

- the Thurston's pullback map $\sigma_{F}: \mathcal{T}_{Z} \rightarrow \mathcal{T}_{Z}$ has a unique fixed point $\tau$;

- the sequence $\left[\phi_{n}\right]$ converges to $\tau$ in the Teichmüller space $\mathcal{T}_{Z}$;

- $\left\{f_{n}\right\}$ converges uniformly to a rational map $f$ on $\widehat{\mathbb{C}}$; and

- $\phi_{n}(Z)$ converges pointwise to a set $X \subset \widehat{\mathbb{C}}$.

Moreover, there is an equivalence $(\phi, \psi)$ between $(F, Z)$ and $(f, X)$ with $\phi, \psi$ : $S^{2} \rightarrow \widehat{\mathbb{C}}$ both representing the fixed point $\tau \in \mathcal{T}_{Z}$.

An easy corollary of the above theorem is that if $Z$ contains more than $\operatorname{deg}(F)+1$ fixed points then $(F, Z)$ is necessarily obstructed. There might be a direct proof of this fact without using Thurston's theorem.

It is easy to see that Theorem 2.2 implies Theorem 2.1. The sequence $\left(\phi_{n}, f_{n}\right)$ appearing in the previous theorem is called Thurston's algorithm for 
the pair $\left(F, \phi_{0}\right)$. Its definition is sketched on the commutative diagram below.

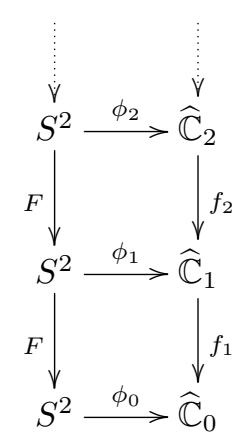

Let us now state without proof a result of McMullen ([McM], Theorem B4) which is closely related to the previous discussion. Again this form is slightly stronger than McMullen's original version but the proof goes through without any trouble.

Theorem 2.3. Let $f: \widehat{\mathbb{C}} \rightarrow \widehat{\mathbb{C}}$ be a rational map (not necessarily postcritically finite), and let $\widehat{Z} \subseteq \widehat{\mathbb{C}}$ be closed (not necessarily finite) with $\mathcal{P}_{f} \subseteq \widehat{Z}$ and $f(\widehat{Z}) \subseteq \widehat{Z}$. Let $\Gamma$ be a $(f, \widehat{Z})$-stable multicurve (defined in a similar way as in the case that $\widehat{Z}$ is finite). Then $\lambda_{\Gamma} \leq 1$. If $\lambda_{\Gamma}=1$, then either $f$ is a $(2,2,2,2)$-map; or $f$ is not postcritically finite, and $\Gamma$ includes a curve that is contained in a Siegel disk or a Herman ring.

\subsection{Classical results from hyperbolic geometry}

In this chapter we will make the following convention on the choice of the multiplicative constant in a hyperbolic metric.

1) The hyperbolic metric on the unit disc $\mathbb{D}$ is $\frac{2|\mathrm{~d} z|}{1-|z|^{2}}$, and on the upper half plane $\mathbb{H}$ it is $\frac{|\mathrm{d} z|}{\Im z}$.

2 ) The modulus of an open annulus $A$ is denoted by $\bmod (A)$, and

$$
\bmod (\{1<|z|<r\})=\frac{\log r}{2 \pi} .
$$

3) For $S$ a hyperbolic Riemann surface and $\gamma$ a closed geodesic on $S$, we use $\ell_{S}(\gamma)$ (or $\ell(\gamma)$ if there is no confusion) to denote the hyperbolic length of $\gamma$. Set $w(\gamma)=-\log \ell(\gamma)$ (one should consider it as a kind of logarithmic width). 4) For any non-peripheral simple closed curve $\gamma$ on $S^{2} \backslash Z$ and any point $\tau \in \mathcal{T}_{Z}$ represented by $\phi: S^{2} \rightarrow \widehat{\mathbb{C}}$, we denote by $\ell(\gamma, \tau)$ the length of the unique simple closed geodesic in $\widehat{\mathbb{C}} \backslash \phi(Z)$ homotopic to $\phi(\gamma)$ and $w(\gamma, \tau)=-\log \ell(\gamma, \tau)$. 
Denote $w(\tau)=\sup w(\gamma, \tau)$ where the supremum is taken over all simple closed geodesics in $S^{2} \backslash Z$.

5) For any constant $C>0$, set

$$
\begin{aligned}
\mathcal{T}_{Z}(C) & =\left\{\tau \in \mathcal{T}_{Z} \mid w(\tau) \leq C\right\} \\
& =\left\{\begin{array}{l|l}
\tau \in \mathcal{T}_{Z} & \begin{array}{l}
-\log \ell(\gamma, \tau) \leq C \text { for every non-peripheral } \\
\text { simple closed curve } \gamma \text { in } S^{2} \backslash Z
\end{array}
\end{array}\right\} .
\end{aligned}
$$

The following result is a version of Wolpert's Lemma which gives an upper bound for ratios of hyperbolic lengths in terms of Teichmüller distances.

Lemma 2.4. Let $\tau_{1}, \tau_{2} \in \mathcal{T}_{Z}$. Assume $d_{\mathcal{T}}\left(\tau_{1}, \tau_{2}\right) \leq D$. Then for any non peripheral simple closed curve $\gamma$ in $S^{2} \backslash Z$,

$$
\left|w\left(\gamma, \tau_{1}\right)-w\left(\gamma, \tau_{2}\right)\right| \leq 2 D
$$

If in addition $\tau_{1} \in \mathcal{T}_{Z}(C)$, then $\tau_{2} \in \mathcal{T}_{Z}(C+2 D)$.

Proof. Let $D^{\prime}>D$ be arbitrary. Let $\phi_{1}, \phi_{2}$ be representatives of $\tau_{1}, \tau_{2}$ respectively. There is a quasi-conformal homeomorphism $h: \phi_{1}\left(S^{2} \backslash Z\right) \rightarrow$ $\phi_{2}\left(S^{2} \backslash Z\right)$ homotopic to $\phi_{2} \circ \phi_{1}^{-1}$ with $\frac{1}{2} \log K(h) \leq D^{\prime}$. Set $S_{1}=\phi_{1}\left(S^{2} \backslash\right.$ $Z)$ and $S_{2}=\phi_{2}\left(S^{2} \backslash Z\right)$. Let $\gamma_{1}$ be a closed geodesic on $S_{1}$ and $\gamma_{2}$ the closed geodesic on $S_{2}$ homotopic to $h\left(\gamma_{1}\right)$. Let $A_{1} \rightarrow S_{1}$ be an annular cover associated to $\gamma_{1}$ and $A_{2} \rightarrow S_{2}$ be an annular cover associated to $\gamma_{2}$. Then

$$
\bmod \left(A_{1}\right)=\frac{\pi}{\ell_{S_{1}}\left(\gamma_{1}\right)} \quad \text { and } \bmod \left(A_{2}\right)=\frac{\pi}{\ell_{S_{2}}\left(\gamma_{2}\right)}
$$

In addition, $h: S_{1} \rightarrow S_{2}$ lifts to a $K(h)$-quasiconformal homeomorphism between $A_{1}$ and $A_{2}$, and according to Grötzsch's inequality,

$$
\bmod \left(A_{1}\right) \leq K(h) \cdot \bmod \left(A_{2}\right) \quad \text { and } \bmod \left(A_{2}\right) \leq K(h) \cdot \bmod \left(A_{1}\right) .
$$

This yields

$$
\left|\log \frac{\ell_{S_{1}}\left(\gamma_{1}\right)}{\ell_{S_{2}}\left(\gamma_{2}\right)}\right|=\left|\log \frac{\bmod \left(A_{1}\right)}{\bmod \left(A_{2}\right)}\right| \leq \log K(h) .
$$

Therefore for any non peripheral simple closed curve $\gamma$ in $S^{2} \backslash Z$,

$$
\left|w\left(\gamma, \tau_{1}\right)-w\left(\gamma, \tau_{2}\right)\right|=\left|\log \ell\left(\gamma, \tau_{1}\right)-\log \ell\left(\gamma, \tau_{2}\right)\right| \leq \log K(h) \leq 2 D^{\prime} .
$$

As $D^{\prime}>D$ is arbitrary, we may replace $D^{\prime}$ by $D$ in the inequality.

Lemma 2.5. Let $S$ be a hyperbolic Riemann surface. 
(1) (short geodesics are simple and disjoint) Let $\gamma_{1}, \gamma_{2}$ be distinct closed geodesics on $S$.

$$
\begin{aligned}
& \ell\left(\gamma_{i}\right)<2 \log (1+\sqrt{2}), i=1,2 \\
& \quad \Longrightarrow \gamma_{1} \cap \gamma_{2}=\emptyset \text { and } \gamma_{1}, \gamma_{2} \text { are simple. }
\end{aligned}
$$

(2) Let $A \subseteq S$ be an open annulus whose equator is homotopic to a simple closed geodesic $\gamma$ on $S$. Then

$$
\bmod A \leq \frac{\pi}{\ell(\gamma)} .
$$

(3) (collar) For any simple closed geodesic $\gamma$ on $S$, there is a canonical annulus $C_{S}(\gamma) \subset S$ whose equator coincides with $\gamma$, with

$$
\bmod C_{S}(\gamma)>\frac{\pi}{\ell(\gamma)}-1
$$

Moreover if two simple closed geodesics $\xi, \eta$ are disjoint, then $C_{S}(\xi)$ and $C_{S}(\eta)$ are disjoint.

Proof. This is a classical result in hyperbolic geometry. Part (3) is attributed to Buser and to Bers ("the collar lemma"). See e.g. Hubbard, [Hu].

Lemma 2.6. (Short geodesics under a forgetful map) Let $S$ be a hyperbolic Riemann surface and $S^{\prime}=S \backslash Q$ with $Q \subset S$ a finite set. Choose $L<$ $2 \log (1+\sqrt{2})$. Set $q=\# Q$. Let $\gamma$ be a simple closed geodesic on $S$. Denote by $\left\{\gamma_{i}^{\prime}\right\}_{i \in I}$ the set of simple closed geodesics on $S^{\prime}$ homotopic to $\gamma$ in $S$ so that the hyperbolic length $\ell_{i}^{\prime}:=\ell_{S^{\prime}}\left(\gamma_{i}^{\prime}\right)$ satisfies $\ell_{i}^{\prime}<L$. Set $\ell=\ell_{S}(\gamma)$. Then

(1) For every $i \in I, \ell_{i}^{\prime} \geq \ell$, and $\# I \leq q+1$ (in particular it is finite).

(2)

$$
\frac{1}{\ell}-\frac{1}{\pi}-\frac{q+1}{L}<\sum_{i \in I} \frac{1}{\ell_{i}^{\prime}}<\frac{1}{\ell}+\frac{q+1}{\pi},
$$

in particular if $I=\emptyset$ then $\frac{1}{\ell}-\frac{1}{\pi}-\frac{q+1}{L}<0$.

Proof. The fact $\ell_{i}^{\prime} \geq \ell$ follows from Schwarz Lemma.

Apply (2.2) to $S^{\prime}$. We know that the $\gamma_{i}^{\prime \prime}$ s are pairwise disjoint. Also, any pair $\gamma_{i}^{\prime}, \gamma_{j}^{\prime}$ enclose an annulus in $S$ (since they are homotopic in $S$ and disjoint) containing at least one point of $Q$ (since they are not homotopic in $S^{\prime}$ ). It follows that there are at most $q+1$ such curves.

It follows from (2.4) that the collars $C_{S^{\prime}}\left(\gamma_{i}^{\prime}\right)$ are pairwise disjoint. There is therefore an open annulus $A \subseteq S$ containing $\bigcup_{i \in I} C_{S^{\prime}}\left(\gamma_{i}^{\prime}\right)$ with equator homotopic to $\gamma$ on $S$. 
The right hand side of (2.5) is trivial if $I=\emptyset$, otherwise,

$$
\begin{gathered}
\sum_{i \in I} \frac{\pi}{\ell_{i}^{\prime}}-(q+1) \stackrel{\#}{\stackrel{\# \leq q+1}{\leq}} \sum_{i \in I}\left(\frac{\pi}{\ell_{i}^{\prime}}-1\right) \stackrel{(2.4)}{<} \sum_{i \in I} \bmod C_{S^{\prime}}\left(\gamma_{i}^{\prime}\right) \\
\stackrel{\text { Grötzsch }}{\leq} \bmod A \stackrel{(2.3)}{\leq} \frac{\pi}{\ell} .
\end{gathered}
$$

We now prove the left hand side inequality of (2.5). We first decompose $C_{S}(\gamma)$ into $t(1 \leq t \leq q+1)$ pairwise disjoint annuli $C_{j}$ such that $\sum_{j=1}^{t} \bmod C_{j}=\bmod C_{S}(\gamma), C_{j} \subset S^{\prime}$, and the core curves of $C_{j}$ are pairwise non-homotopic in $S^{\prime}$. For each $j$, let $\delta_{j}$ be the geodesic on $S^{\prime}$ homotopic in $S^{\prime}$ to the core curve $C_{j}$.

We have then

$$
\begin{aligned}
\frac{\pi}{\ell}-1 \stackrel{(2.4)}{<} \bmod C_{S}(\gamma) & =\sum_{j=1}^{t} \bmod C_{j}=\left(\sum_{\bmod C_{j} \leq \frac{\pi}{L}}+\sum_{\bmod C_{j}>\frac{\pi}{L}}\right) \bmod C_{j} \\
& \leq \frac{(q+1) \pi}{L}+\sum_{\bmod C_{j}>\frac{\pi}{L}} \bmod C_{j} \stackrel{(2.3)}{\leq} \frac{(q+1) \pi}{L}+\sum_{\bmod C_{j}>\frac{\pi}{L}} \frac{\pi}{\ell_{S^{\prime}}\left(\delta_{j}\right)}
\end{aligned}
$$

Assume that the index set of the rightmost term is non empty. Then $\ell_{S^{\prime}}\left(\delta_{j}\right)<L$ so $\delta_{j}=\gamma_{i}^{\prime}$ for some $i \in I$, in particular $I \neq \emptyset$. In this case

$$
\frac{\pi}{\ell}-1<\frac{(q+1) \pi}{L}+\sum_{i \in I} \frac{\pi}{\ell_{i}^{\prime}} .
$$

If $I=\emptyset$, then necessarily no $C_{j}$ satisfies $\bmod C_{j}>\frac{\pi}{L}$ and we have

$$
\frac{\pi}{\ell}-1<\frac{(q+1) \pi}{L} .
$$

The left hand inequality of (2.5) is now proved.

\subsection{From $\mathcal{T}_{Z}$ to Rat $_{d}$}

From now on, we fix three points $x_{0}, x_{1}, x_{2}$ in $Z \subseteq F^{-1}(Z)$. A point $\tau \in \mathcal{T}_{Z}$ may be represented by a homeomorphism $\phi: S^{2} \rightarrow \widehat{\mathbb{C}}$ sending $x_{0}, x_{1}, x_{2}$ to respectively $0,1, \infty$. Its restriction $\phi_{\tau}: Z \rightarrow \widehat{\mathbb{C}}$ only depends on $\tau$. Similarly, $\varsigma_{F}(\tau) \in \mathcal{T}_{F^{-1}(Z)}$ may be represented by a homeomorphism $\psi: S^{2} \rightarrow \widehat{\mathbb{C}}$ sending $x_{0}, x_{1}, x_{2}$ to respectively $0,1, \infty$, so that $f=\phi \circ F \circ \psi^{-1}$ is a rational map. The restriction $\psi_{\tau}: F^{-1}(Z) \rightarrow \widehat{\mathbb{C}}$ of $\psi$ and the rational map $f$ only depend on $\tau$. Indeed, if $d_{x}$ stands for the local degree of $F$ at $x \in S^{2}$, then

$$
f=f_{\tau}=\lambda_{\tau} \cdot P_{\tau} / Q_{\tau}
$$


where

$$
P_{\tau}(z)=\prod_{\substack{x \in F-1\left(x_{0}\right) \\ x \neq x_{2}}}\left(z-\psi_{\tau}(x)\right)^{d_{x}}, \quad Q_{\tau}(z)=\prod_{\substack{x \in F^{-1}\left(x_{2}\right) \\ x \neq x_{2}}}\left(z-\psi_{\tau}(x)\right)^{d_{x}}
$$

and $\lambda_{\tau}$ is the value taken by $Q_{\tau} / P_{\tau}$ at any point of $\psi_{\tau}\left(F^{-1}\left(x_{1}\right)\right)$. Since $\varsigma_{F}: \mathcal{T}_{Z} \rightarrow \mathcal{T}_{F^{-1}(Z)}$ is analytic, the map

$$
\mathcal{T}_{Z} \ni \tau \mapsto\left(f_{\tau}, \phi_{\tau}, \psi_{\tau}\right) \in \operatorname{Rat}_{d} \times(\widehat{\mathbb{C}})^{Z} \times(\widehat{\mathbb{C}})^{F^{-1}(Z)}
$$

is analytic.

It is true, although not elementary, that the image of $\mathcal{T}_{Z}$ under the map $\tau \mapsto f_{\tau}$ is closed in Rat $_{d}$. We shall circumvent the difficulties by introducing the following space. We shall denote by $\mathcal{R}_{Z, F}$ the set of triples

$$
(f, \phi, \psi) \in \operatorname{Rat}_{d} \times(\widehat{\mathbb{C}})^{Z} \times(\widehat{\mathbb{C}})^{F^{-1}(Z)}
$$

such that

- $\phi$ and $\psi$ are injections sending $x_{0}, x_{1}, x_{2}$ to respectively $0,1, \infty$,

- $\phi \circ F=f \circ \psi$ on $F^{-1}(Z)$ and

- the local degree of $F$ at $x$ is equal to that of $f$ at $\psi(x)$ for all $x \in F^{-1}(Z)$. In particular, setting $Y=\phi(Z)$ and $X=\psi\left(F^{-1}(Z)\right)=f^{-1}(Y)$, we have the following commutative diagram:

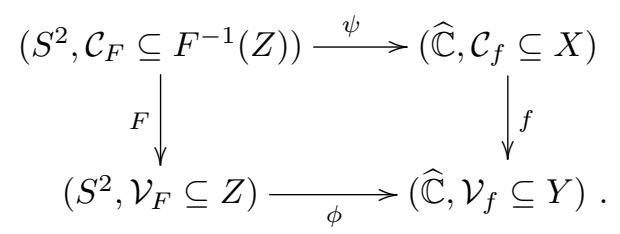

Let $d_{\widehat{\mathbb{C}}}$ stand for the spherical distance in $\widehat{\mathbb{C}}$. Given $c>0$, we shall denote by $\mathcal{R}_{Z, F}(c)$ the subset of $\mathcal{R}_{Z, F}$ consisting of those triples $(f, \phi, \psi)$ for which $d_{\widehat{\mathbb{C}}}\left(z_{1}, z_{2}\right) \geq c$ for any pair of distinct points $z_{1} \neq z_{2}$ in $\psi\left(F^{-1}(Z)\right)$.

Lemma 2.7. For all $c>0$, the set $\mathcal{R}_{Z, F}(c)$ is a compact subset of $\mathcal{R}_{Z, F}$. For all $C>0$ there exists $c>0$ such that

$$
\tau \in \mathcal{T}_{Z}(C) \quad \Longrightarrow \quad\left(f_{\tau}, \phi_{\tau}, \psi_{\tau}\right) \in \mathcal{R}_{Z, F}(c)
$$

Proof. Let $\left(f_{n}, \phi_{n}, \psi_{n}\right)$ be a sequence of triples in $\mathcal{R}_{Z, F}(c)$. Set $Y_{n}=\phi_{n}(Z)$ and $X_{n}=\psi_{n}\left(F^{-1}(Z)\right)=f_{n}^{-1}\left(Y_{n}\right)$. Since $\widehat{\mathbb{C}}$ is compact, extracting a subsequence if necessary, we may assume that the sequences $\left(\phi_{n}: Z \rightarrow Y_{n}\right)$ and $\left(\psi_{n}: F^{-1}(Z) \rightarrow X_{n}\right)$ converge respectively to maps $\phi: Z \rightarrow Y$ and $\psi: F^{-1}(Z) \rightarrow X$ for some finite sets $X, Y \subset \widehat{\mathbb{C}}$. Since the spherical distance between distinct points in $X_{n}$ is at least $c>0$, the limit $\psi: Z \rightarrow X$ is a 
bijection and the spherical distance between distinct points in $X$ is at least $c$. The sequence of rational maps $f_{n}$ converges to $f=\lambda \cdot P / Q$ where

$$
P(z)=\prod_{\substack{x \in F-1\left(x_{0}\right) \\ x \neq x_{2}}}(z-\psi(x))^{d_{x}}, \quad Q(z)=\prod_{\substack{x \in F-1 \\ x \neq x_{2}}}(z-\psi(x))^{d_{x}}
$$

and $\lambda$ is the value taken by $Q / P$ at any point of $\psi\left(F^{-1}\left(x_{1}\right)\right)$. If $x \in F^{-1}(Z)$, then

$$
f \circ \psi(x)=\lim f_{n} \circ \psi_{n}(x)=\lim \phi_{n} \circ F(x)=\phi \circ F(x) .
$$

The local degree of $f$ at a point $\psi(x)$ is at least $d_{x}$, and for all $y \in Y$, the number of preimages of $y$ by $f$, counting multiplicities is

$$
\begin{aligned}
d & =\sum_{x \in(\phi \circ F)^{-1}(y)} \operatorname{deg}(f, \psi(x)) \geq \sum_{x \in(\phi \circ F)^{-1}(y)} d_{x} \\
& \geq \sum_{z \in \phi^{-1}(y)} \sum_{x \in F^{-1}(z)} d_{x}=d \cdot \# \phi^{-1}(y) .
\end{aligned}
$$

Thus, $\# \phi^{-1}(y)=1$, i.e. $\phi$ is injective, and the local degree of $f$ at $\psi(x)$ is $d_{x}$. All this shows that $(f, \phi, \psi) \in \mathcal{R}_{Z, F}(c)$.

This proves that $\mathcal{R}_{Z, F}(c)$ is a compact subset of $\operatorname{Rat}_{d} \times(\widehat{\mathbb{C}})^{Z} \times(\widehat{\mathbb{C}})^{F^{-1}(Z)}$.

Let us now prove that the image of $\mathcal{T}_{Z}(C)$ is contained in $\mathcal{R}_{Z, F}(c)$ for some $c>0$. Set $Y_{\tau}=\phi_{\tau}(Z)$ and $X_{\tau}=\psi_{\tau}\left(F^{-1}(Z)\right)=f_{\tau}^{-1}\left(Y_{\tau}\right)$. By definition of $\mathcal{T}_{Z}(C)$, the length of any simple closed geodesic $\gamma \in \widehat{\mathbb{C}} \backslash Y_{\tau}$ is bounded from below by $e^{-C}$. Since $Y_{\tau}$ contains the critical values of $f_{\tau}$, the map $f_{\tau}: \widehat{\mathbb{C}} \backslash X_{\tau} \rightarrow \widehat{\mathbb{C}} \backslash Y_{\tau}$ is a covering. It follows that the length of any simple closed geodesic $\delta \in \widehat{\mathbb{C}} \backslash X_{\tau}$ is bounded from below by $e^{-C}$. As a consequence, as $\tau$ ranges in $\mathcal{T}_{Z}(C)$ and $x, y$ range in $F^{-1}(Z)$ with $x \neq y$, the spherical distance between $\psi_{\tau}(x)$ and $\psi_{\tau}(y)$ is uniformly bounded away from 0 as required.

\subsection{Contraction of Thurston's pullback maps}

Let $F: S^{2} \rightarrow S^{2}$ be a branched covering of degree $d \geq 2$ with a finite postcritical set $\mathcal{P}_{F}$. Let $Z \subset \widehat{\mathbb{C}}$ be a finite set with $\# Z \geq 4, \mathcal{P}_{F} \subseteq Z$ and $F(Z) \subseteq Z$. Setting $X=Y=Z$, the conditions in Section 1.4 are satisfied and thus, the Thurston's pullback map $\sigma_{F}: \mathcal{T}_{Z} \rightarrow \mathcal{T}_{Z}$ is well defined. From now on, we set

$$
k=\# Z \text { and } G=F^{\circ k} \text {. }
$$

Recall that the tangent space to $\mathcal{T}_{Z}$ at some point $\tau$ represented by $\psi$ : $S^{2} \rightarrow \widehat{\mathbb{C}}$ is equipped with the dual norm:

$$
\forall \nu \in T_{\tau} \mathcal{T}_{Z}, \quad\|\nu\|=\sup _{\substack{q \in \mathcal{Q}(\psi(Z)) \\\|q\| \leq 1}}|\langle q, \nu\rangle|
$$


We will now show that $\sigma_{F}^{\circ k}$ is contracting, and even uniformly contracting on $\mathcal{T}_{Z}(C)$ for $C>0$. It will be useful to notice that $\sigma_{F}^{\circ k}=\sigma_{G}$. Indeed, assume $\tau$ is a point in $\mathcal{T}_{Z}$, and for $i \in[0, k]$, let $\phi_{i}: S^{2} \rightarrow \widehat{\mathbb{C}}$ be a homeomorphism representing $\sigma_{F}^{\circ i}(\tau)$ so that $f_{i}=\phi_{i} \circ F \circ \phi_{i+1}^{-1}: \widehat{\mathbb{C}} \rightarrow \widehat{\mathbb{C}}$ is a rational map for $i \in[0, k-1]$. Then, we have the following commutative diagram with $Z_{i}=\phi_{i}(Z)$ :

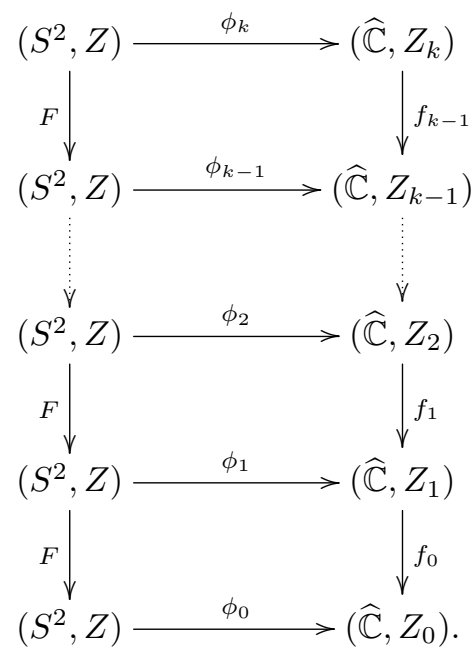

Set $\phi=\phi_{0}, \psi=\phi_{k}$ and $g=f_{0} \circ f_{1} \circ \cdots \circ f_{k-1}$. Then, the commutative diagram

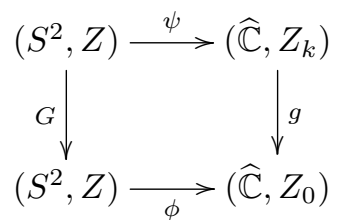

shows that

$$
\sigma_{F}^{\circ k}(\tau)=[\psi]=\sigma_{G}([\phi])=\sigma_{G}(\tau)
$$

Lemma 2.8. If there is a set $X \subseteq Z$ such that $\# X \geq 4$ and $G^{-1}(X) \subseteq Z \cup \mathcal{C}_{G}$, then $F: S^{2} \rightarrow S^{2}$ is a $(2,2,2,2)$-map.

Proof. Define recursively

$$
X_{0}=X \quad \text { and } \quad X_{i+1}=F^{-1}\left(X_{i}\right) \backslash \mathcal{C}_{F},
$$

so that $X_{k}=G^{-1}(X) \backslash \mathcal{C}_{G} \subseteq Z$. In particular, \# $X_{k} \leq \# Z=k$. 
Since $F^{-1}\left(X_{i}\right) \subseteq X_{i+1} \cup \mathcal{C}_{F}$, we have the following inequalities (compare with (1.6)):

$$
\# X_{i+1}+(2 d-2) \geq \# X_{i+1}+\# \mathcal{C}_{F} \geq \# F^{-1}\left(X_{i}\right) \geq d \cdot \# X_{i}-(2 d-2) .
$$

This implies $\# X_{i+1}-4 \geq d \cdot\left(\# X_{i}-4\right)$. In particular,

$$
\begin{array}{r}
d^{k-4}>k-4 \geq \# X_{k}-4 \geq d^{k-4} \cdot\left(\# X_{4}-4\right) \geq \cdots \\
\geq d^{k-1} \cdot\left(\# X_{1}-4\right) \geq d^{k} \cdot\left(\# X_{0}-4\right) \geq 0 .
\end{array}
$$

As a consequence, $\# X_{i}=4$ for $i=0,1,2,3,4$ and inequalities (2.7) must be equalities for $i=0,1,2,3$ :

- $\# \mathcal{C}_{F}=2 d-2$, thus the critical points of $F$ are simple.

- $\# X_{i+1}+\# \mathcal{C}_{F}=\# F^{-1}\left(X_{i}\right)$, thus $\mathcal{C}_{F} \cap X_{i+1}=\emptyset$. In particular, $\mathcal{C}_{F} \cap X_{1}=$ $\emptyset$.

- \# $F^{-1}\left(X_{i}\right)=d \cdot \# X_{i}-(2 d-2)$, thus $\mathcal{V}_{F} \subseteq X_{i}$. In particular, $\mathcal{V}_{F^{\circ 3}}=\mathcal{V}_{F} \cup F\left(\mathcal{V}_{F}\right) \cup F^{\circ 2}\left(\mathcal{V}_{F}\right) \subseteq X_{1}$ and $\mathcal{V}_{F^{\circ 4}}=F\left(\mathcal{V}_{F^{\circ 3}}\right) \cup F^{\circ 3}\left(\mathcal{V}_{F}\right) \subseteq X_{0}$

We now claim that $X_{1}=\mathcal{P}_{F}$. Indeed

- $X_{1} \subseteq \mathcal{P}_{F}$ since otherwise a point in $X_{1} \backslash \mathcal{P}_{F}$ would have $d^{3}>4$ preimages in $X_{4}$ whereas $\# X_{4}=4$.

- $\mathcal{P}_{F} \subseteq X_{1}$ since

$$
\mathcal{V}_{F} \subseteq \mathcal{V}_{F^{\circ 2}} \subseteq \mathcal{V}_{F^{\circ 3}} \subseteq \mathcal{V}_{F^{\circ 4}} \subseteq X_{0}
$$

so that

$$
2 \leq \# \mathcal{V}_{F} \leq \# \mathcal{V}_{F^{\circ 2}} \leq \# \mathcal{V}_{F^{\circ 3}} \leq \# \mathcal{V}_{F^{\circ 4}} \leq 4
$$

which forces the non-decreasing sequence $\mathcal{V}_{F^{\circ i}}$ to stabilize: there exists $i_{0} \leq 3$ such that $\mathcal{V}_{F^{\circ i}}=\mathcal{V}_{F^{\circ i_{0}}}$ for $i \geq i_{0}$. We then have $\mathcal{P}_{F}=\mathcal{V}_{F^{\circ i_{0}}}=$ $\mathcal{V}_{F^{\circ 3}} \subseteq X_{1}$.

Summarizing, we see that $\mathcal{P}_{F}=X_{1}$ has cardinality $4, \mathcal{C}_{F} \cap \mathcal{P}_{F}=\mathcal{C}_{F} \cap X_{1}=\emptyset$ and all the critical points of $F$ are simple. Thus, $F$ is a $(2,2,2,2)$-map.

Lemma 2.9 (Contraction). If $F: S^{2} \rightarrow S^{2}$ is not a $(2,2,2,2)-m a p$, then $\left\|D_{\tau} \sigma_{F}^{\circ k}\right\|<1$ (where $k=\# Z$ ) for any $\tau \in \mathcal{T}_{Z}{ }^{1}$

Proof. A point $\tau \in \mathcal{T}_{Z}$ yields a triple $\left(g_{\tau}, \phi_{\tau}, \psi_{\tau}\right) \in \mathcal{R}_{Z, G}$ such that $g_{\tau} \circ \psi_{\tau}=$ $\phi_{\tau} \circ G$ on $G^{-1}(Z)$. The norm of the linear map $D_{\tau} \sigma_{F}^{\circ k}=D_{\tau} \sigma_{G}$ is equal to the norm of its adjoint $\left(g_{\tau}\right)_{*}: \mathcal{Q}\left(\psi_{\tau}(Z)\right) \rightarrow \mathcal{Q}\left(\phi_{\tau}(Z)\right)$. The result is a consequence of the following more general Lemma.

\footnotetext{
${ }^{1}$ It is known that in the classical version of Thurston's theorem where $Z=\mathcal{P}_{F}$, one may choose $k=2$. In the general version, it is possible to prove that we may choose $k \geq 2$ such that $d^{k-2}>\#\left(Z \backslash \mathcal{P}_{F}\right)$.
} 
Lemma 2.10. Assume $F: S^{2} \rightarrow S^{2}$ is not $(2,2,2,2)$-map and $(g, \phi, \psi) \in$ $\mathcal{R}_{Z, G}$. Then, $g_{*}: \mathcal{Q}(\psi(Z)) \rightarrow \mathcal{Q}(\phi(Z))$ has norm strictly less than 1 .

Proof. Due to Lemma 1.6 we know already $\left\|g_{*}\right\| \leq 1$, with equality if and only if there is a non-zero $q \in \mathcal{Q}(\psi(Z))$ such that $q=d^{-k} g^{*}\left(g_{*} q\right)$.

Assume by contradiction that $\left\|g_{*}\right\|=1$. Let $Y \subseteq \phi(Z)$ be the set of poles of $g_{*} q$. Then, every point of $g^{-1}(Y)$ is either a pole of $q$, or a critical point of g. So, $g^{-1}(Y) \subseteq \psi(Z) \cup \mathcal{C}_{g}$. As a consequence, $X=\phi^{-1}(Y)$ satisfies $X \subseteq Z$ and $G^{-1}(X) \subseteq Z \cup \mathcal{C}_{G}$. According to Lemma 2.8, this contradicts the fact that $F$ is not a $(2,2,2,2)$-map.

Lemma 2.11 (Uniform contraction on $\mathcal{T}_{Z}(C)$ ). If $F$ is not a $(2,2,2,2)$-map, then for each $C>0$, there is $\lambda<1$ such that $\left\|D_{\tau} \sigma_{F}^{\circ k}\right\| \leq \lambda$ for all $\tau \in \mathcal{T}_{Z}(C)$.

Proof. We proceed by contradiction and assume we can find a sequence $\tau_{n} \in \mathcal{T}_{Z}(C)$ such that $\left\|D_{\tau_{n}} \sigma_{F}^{\circ k}\right\|$ tends to 1 as $n$ tends to $\infty$. Consider the corresponding sequence of triples $\left(g_{n}, \phi_{n}, \psi_{n}\right) \in \mathcal{R}_{Z, G}$. Set $X_{n}=\psi_{n}(Z)$ and $Y_{n}=\phi_{n}(Z)$. The norm $\left\|D_{\tau_{n}} \sigma_{F}^{\circ k}\right\|$ is equal to the norm of $\left(g_{n}\right)_{*}: \mathcal{Q}\left(X_{n}\right) \rightarrow$ $\mathcal{Q}\left(Y_{n}\right)$. Thus, we can find a sequence of quadratic differentials $q_{n} \in \mathcal{Q}\left(X_{n}\right)$ of norm 1 so that $\left\|\left(g_{n}\right)_{*} q_{n}\right\|$ tends to 1 as $n$ tends to $\infty$.

According to Lemma 2.7, this sequence belongs to a compact subset of $\mathcal{R}_{Z, G}$. So, extracting a subsequence if necessary, we may assume that the triple $\left(g_{n}, \phi_{n}, \psi_{n}\right)$ converges to $(g, \phi, \psi) \in \mathcal{R}_{Z, G}$. According to the previous lemma, the norm of $g_{*}: \mathcal{Q}(X) \rightarrow \mathcal{Q}(Y)$ is less than 1 .

The poles of the quadratic differentials $q_{n}$ are simple and stay uniformly away from each other for the spherical distance. It follows that we may extract a further subsequence so that $q_{n}$ converges locally uniformly outside $X$ to some $q \in \mathcal{Q}(X)$ of norm 1. The sequence of quadratic differentials $\left(g_{n}\right)_{*} q_{n}$ then converges locally uniformly to $g_{*} q \in \mathcal{Q}(Y)$ outside $Y$. Since the poles of $\left(g_{n}\right)_{*} q_{n}$ are in $Y_{n}$, they remain uniformly away from each other for the spherical distance. As a consequence, $\left\|g_{*} q\right\|=\lim \left\|\left(g_{n}\right)_{*} q_{n}\right\|=1=\|q\|$. This contradicts the previous observation that $\left\|g_{*}\right\|<1$.

\subsection{Proof of Theorem 2.2}

Proposition 2.12. (short geodesics do not become shorter) Assume that $(F, Z)$ has no Thurston obstructions. Given $\tau_{0} \in \mathcal{T}_{Z}$, set $\tau_{n}=\sigma_{F}^{\circ n}\left(\tau_{0}\right)$. Then there is a positive integer $m$ depending only on $\operatorname{deg}(F)$ and $\# Z$, a positive constant $C$ depending only on $\operatorname{deg}(F), \# Z$ and $d_{\mathcal{T}}\left(\tau_{0}, \tau_{1}\right)$, such that:

$$
\forall n \geq 0, \quad w\left(\tau_{n}\right)>C \quad \Rightarrow \quad w\left(\tau_{n+m}\right)<w\left(\tau_{n}\right) .
$$

We will postpone the proof of this proposition to Section 2.7. 


\section{Proof of Theorem 2.2 assuming proposition 2.12.}

Given $\tau_{0} \in \mathcal{T}_{Z}$, set $\tau_{n}=\sigma_{F}^{\circ n}\left(\tau_{0}\right)$ and $D=d_{\mathcal{T}}\left(\tau_{0}, \tau_{1}\right)$. Let $\delta$ be a geodesic of $\mathcal{T}_{Z}$ connecting $\tau_{0}$ and $\tau_{1}$ and for $n \geq 0$, set $\delta_{n}=\sigma_{F}^{\circ n}(\delta)$. According to Lemma 2.4, $\sigma_{F}: \mathcal{T}_{Z} \rightarrow \mathcal{T}_{Z}$ is contracting and so, for all $n \geq 0$, we have length $\left(\delta_{n+1}\right) \leq \operatorname{length}\left(\delta_{n}\right)$. It then follows from Lemma 2.9 that

$$
\forall n \geq 0, d_{\mathcal{T}}\left(\tau_{n}, \tau_{n+1}\right) \leq d_{\mathcal{T}}\left(\tau_{0}, \tau_{1}\right) \leq D \text { and } w\left(\tau_{n+1}\right) \leq w\left(\tau_{n}\right)+2 D .
$$

Let $m$ and $C$ be given by Proposition 2.12. Set $C_{1}=\max \left(C, w\left(\tau_{0}\right)\right)$. We claim that the sequence $\left(\tau_{n}\right)_{n \geq 0}$ remains in $\mathcal{T}_{Z}\left(C_{1}+2 m D\right)$. Indeed, for $n \geq 0$, let $j_{n} \in[0, n]$ be the largest integer $j$ such that $\tau_{j} \in \mathcal{T}_{Z}\left(C_{1}\right)$. If $j_{n}=n$, then we are done. Otherwise, let us write $n=\left(j_{n}+1\right)+q m+r$ with $0 \leq r<m$. For $j \in\left[j_{n}+1, n\right]$, we have $w\left(\tau_{j}\right)>C_{1} \geq C$. It follows from Proposition 2.12 and Lemma 2.4 that

$$
w\left(\tau_{n}\right) \leq w\left(\tau_{j_{n}+1+r}\right) \leq w\left(\tau_{j_{n}}\right)+(r+1) \cdot 2 D \leq C_{1}+m \cdot 2 D .
$$

Set $C_{2}=C_{1}+(m+1) \cdot 2 D$. According to Lemma 2.4, $\delta_{n} \subset \mathcal{T}_{Z}\left(C_{2}\right)$ for all $n \geq 0$. Set $k=\# Z$. By Lemma 2.11, there is a constant $\lambda<1$ such that $\left\|D_{\tau} \sigma_{F}^{\circ k}\right\|<\lambda$ for any $\tau \in \mathcal{T}_{Z}(C)$. It follows that, for any $n \geq 0$ and any $1 \leq j \leq k$,

$$
d_{\mathcal{T}}\left(\tau_{n k}, \tau_{n k+j}\right) \leq \lambda^{n} d_{\mathcal{T}}\left(\tau_{0}, \tau_{j}\right) \leq j D \lambda^{n} \leq k D \lambda^{n} .
$$

Therefore $\left(\tau_{n}\right)_{n \geq 0}$ is a Cauchy sequence in $\mathcal{T}_{Z}$ and hence converges to a fixed point $\tau$ in $\mathcal{T}_{Z}$.

We now prove that the fixed point is unique, independent of the choice of $\tau_{0}$. Indeed, let $\tau$ and $\tau^{\prime}$ be two fixed points of $\sigma_{F}$. Let $\delta$ be the geodesic joining $\tau$ and $\tau^{\prime}$. Recall that $k=\# Z$. Then $\sigma_{F}^{\circ k}(\delta)$ is a curve joining $\tau$ and $\tau^{\prime}$ and its length is less that that of $\delta$, which contradicts the fact that $\delta$ is the shortest curve joining $\tau$ and $\tau^{\prime}$.

Finally, as $\tau_{n} \in \mathcal{T}_{Z}$ tends to $\tau \in \mathcal{T}_{Z}$, the sequence $\left(f_{\tau_{n}}, \phi_{\tau_{n}}, \psi_{\tau_{n}}\right) \in \mathcal{R}_{Z, F}$ tends to $\left(f_{\tau}, \phi_{\tau}, \psi_{\tau}\right)$. This shows that if $\phi_{0}: S^{2} \rightarrow \widehat{\mathbb{C}}$ is an orientation preserving homeomorphism sending $x_{0}, x_{1}, x_{2}$ to $0,1, \infty$ and if $\left(\phi_{n}, f_{n}\right)$ is defined recursively so that $\phi_{n}: S^{2} \rightarrow \widehat{\mathbb{C}}$ is a homeomorphism sending $x_{0}, x_{1}, x_{2}$ to $0,1, \infty$ and so that the map $f_{n}=\phi_{n-1} \circ F \circ \phi_{n}^{-1}$ is a rational map, then

- $f_{n}=f_{\tau_{n}}$ converges to $f_{\tau}$ and

- $\phi_{n}(Z)=\phi_{\tau_{n}}(Z)$ converges pointwise to a set $X \subset \widehat{\mathbb{C}}$.

Since $\sigma_{F}(\tau)=\tau$ the bijection $\phi_{\tau}: Z \rightarrow X$ coincides with the bijection $\psi_{\tau}$ : $Z \rightarrow X$. It follows that $f_{\tau}(X) \subseteq X$ and that $f_{\tau}$ is postcritically finite with $\mathcal{P}_{f_{\tau}} \subseteq X$.

Finally, let $\phi: S^{2} \rightarrow \widehat{\mathbb{C}}$ be the homeomorphism representing $\tau$ sending $x_{0}, x_{1}, x_{2}$ to $0,1, \infty$. Let $\psi: S^{2} \rightarrow \widehat{\mathbb{C}}$ be the homeomorphism representing $\sigma_{F}(\tau)=\tau$ sending $x_{0}, x_{1}, x_{2}$ to $0,1, \infty$ with $f_{\tau} \circ \psi=\phi \circ F$. Then, $(\phi, \psi)$ is an equivalence between $(F, Z)$ and $(f, X)$. 


\subsection{Proof of Proposition 2.12.}

Notice that from the definition of the transition matrix, given a degree $d$, and an integer $p$, there are only finitely many possible transition matrices $F_{\Gamma}$ with $F$ of degree $d$ and $\Gamma$ of size at most $p-3$. Therefore there are finitely many such matrices with leading eigenvalue $\lambda_{\Gamma}<1$. The integer $m$, depending only on $d$ and $p$, is chosen so that every such matrix $F_{\Gamma}$ with $\lambda_{\Gamma}<1$ satisfies $\left\|F_{\Gamma}^{m}\right\|<1 / 2$, where $\|\cdot\|$ is relative to the sup-norm of $\mathbb{R}^{\Gamma}$ (this is possible due to the spectral radius formula $\left\|F_{\Gamma}^{n}\right\|^{1 / n} \underset{n \rightarrow \infty}{\longrightarrow} \lambda_{\Gamma}$ ).

Set $A=-\log (2 \log (\sqrt{2}+1))$ and $D=d_{\mathcal{T}}\left(\tau_{0}, \tau_{1}\right)$. We choose at first any $J>m(\log d+2 D)$, and set $B=(p-3) J+A$.

For the moment choose any $C>B$ and assume $w\left(\tau_{n}\right)>C$ for some $n \geq 0$. We want to show that $w\left(\tau_{n+m}\right)<w\left(\tau_{n}\right)$, up to a further adjustment of $C$.

Let $\phi: S^{2} \rightarrow \widehat{\mathbb{C}}$ represent $\tau_{n}$. Set $P=\phi(Z)$ and

$$
L_{n}=\left\{w\left(\gamma, \tau_{n}\right) \mid \gamma \text { a non-peripheral Jordan curve on } S^{2} \backslash Z\right\} .
$$

Now let $] a, b\left[\right.$ be the leftmost gap in $\left[A,+\infty\left[\backslash L_{n}\right.\right.$ of length $J$. Set

$$
\Gamma=\left\{\begin{array}{l}
\gamma \mid \begin{array}{l}
\gamma \text { a non-peripheral Jordan curve on } S^{2} \backslash Z \\
\text { with } \left.w\left(\gamma, \tau_{n}\right) \in\right] a,+\infty[
\end{array}
\end{array}\right\} .
$$

Then $w\left(\gamma, \tau_{n}\right) \geq b$ for $\gamma \in \Gamma$. By Lemma 2.5, the set of $\gamma$ with $w\left(\gamma, \tau_{n}\right)>A$ consists of pairwise disjoint non-peripheral simple closed curves in $S^{2} \backslash Z$. But $Z$ consists of exactly $p$ points. It follows that there are at most $p-3$ elements of $L_{n}$ greater than $A$. By assumption $w\left(\tau_{n}\right)>C>B=(p-3) J+A$. So at least one element of $L_{n}$ is greater than $(p-3) J+A$. It follows that $b<B$ and $\Gamma \neq \emptyset$.

Claim (a). The multicurve $\Gamma$ is $(F, Z)$-stable.

Proof. For this we will only use the fact that $J>\log d+2 D$.

Let $\varphi$ be a representative of $\tau_{n+1}=\sigma_{F}\left(\tau_{n}\right)$ such that $f=\phi \circ F \circ \varphi^{-1}$ is a rational map. Set $T^{\prime}=\varphi(Z)$ and $T^{\prime \prime}=\varphi\left(F^{-1}(Z)\right)=f^{-1}(P)$. Given $\gamma \in \Gamma$, let $\eta$ be a non-peripheral Jordan curve in $F^{-1}(\gamma)$. Let $\xi^{\prime}$ (respectively $\xi^{\prime \prime}$ ) be the geodesic homotopic to $\varphi(\eta)$ in $\widehat{\mathbb{C}} \backslash T^{\prime}$ (respectively in $\widehat{\mathbb{C}} \backslash T^{\prime \prime}$ ). Since $f: \widehat{\mathbb{C}} \backslash T^{\prime \prime} \rightarrow \widehat{\mathbb{C}} \backslash P$ is a holomorphic covering, and since $T^{\prime} \subseteq T^{\prime \prime}$, we have

$$
\ell_{\widehat{\mathbb{C}} \backslash T^{\prime \prime}}\left(\xi^{\prime \prime}\right)=\operatorname{deg}(F: \eta \rightarrow \gamma) \cdot \ell_{\widehat{\mathbb{C}} \backslash P}\left(f\left(\xi^{\prime \prime}\right)\right)=\operatorname{deg}(F: \eta \rightarrow \gamma) \cdot \ell\left(\gamma, \tau_{n}\right)
$$

and

$$
\ell_{\widehat{\mathbb{C}} \backslash T^{\prime \prime}}\left(\xi^{\prime \prime}\right) \geq \ell_{\widehat{\mathbb{C}} \backslash T^{\prime}}\left(\xi^{\prime \prime}\right) \geq \ell_{\widehat{\mathbb{C}} \backslash T^{\prime}}\left(\xi^{\prime}\right)=\ell\left(\eta, \tau_{n+1}\right)
$$

Thus

$$
w\left(\eta, \tau_{n+1}\right) \geq w\left(\gamma, \tau_{n}\right)-\log \operatorname{deg}(F: \eta \rightarrow \gamma) \geq w\left(\gamma, \tau_{n}\right)-\log d \geq b-\log d .
$$


By Lemma 2.4, we have $\left|w\left(\eta, \tau_{n+1}\right)-w\left(\eta, \tau_{n}\right)\right| \leq 2 D$. Thus

$$
w\left(\eta, \tau_{n}\right) \geq b-\log d-2 D>a
$$

since $b-a=J>\log d+2 D$. This shows that $\eta$ is homotopic rel $Z$ to a curve in $\Gamma$. That is, $\Gamma$ is an $(F, Z)$-stable multicurve. This ends the proof of Claim (a).

Set $G=F^{\circ m}$. Let $\psi$ be a representative of $\tau_{n+m}$ such that $g=\phi \circ G \circ \psi^{-1}$ is a rational map of degree $d^{m}$. Set $P^{\prime}=\psi(Z)$ and $P^{\prime \prime}=\psi\left(G^{-1}(Z)\right)=g^{-1}(P)$. Then $P^{\prime} \subseteq P^{\prime \prime}$.

Claim (b). Every simple closed geodesic in $\widehat{\mathbb{C}} \backslash P^{\prime \prime}$ of length less than $d^{m} \cdot e^{-b}$ is homotopic (rel $\left.P^{\prime \prime}\right)$ to a component of $g^{-1} \circ \phi(\gamma)$ for some unique choice of $\gamma \in \Gamma$.

Proof. Let $\beta$ be a simple closed geodesic in $\widehat{\mathbb{C}} \backslash P^{\prime \prime}$ of length less than $d^{m} \cdot e^{-b}$. Then $g(\beta)$ is a simple closed geodesic in $\widehat{\mathbb{C}} \backslash P$ with length less than $e^{-b}$, that is, $w(g(\beta)) \geq b$. Thus $g(\beta)$ is homotopic, rel $P$, to $\phi(\gamma)$ for some unique choice of $\gamma \in \Gamma$. The critical values of $g$ are contained in $P$. We may then lift the homotopy by $g$ to get Claim (b).

Set $L=d^{m} \cdot e^{-B}$. Note that $L$ depends only on $p, d$ and $D$. Let $\Gamma=$ $\left\{\gamma_{1}, \cdots, \gamma_{s}\right\}$ be the non-empty $(F, Z)$-stable multicurve defined above. Define $v, v^{\prime} \in \mathbb{R}^{\Gamma}$ by

$$
v_{i}=\frac{1}{\ell\left(\gamma_{i}, \tau_{n}\right)} \quad \text { and } \quad v_{i}^{\prime}=\frac{1}{\ell\left(\gamma_{i}, \tau_{n+m}\right)} .
$$

Set $S=\widehat{\mathbb{C}} \backslash P^{\prime}$ and $Q=P^{\prime \prime} \backslash P^{\prime}$. Set $q=\# Q=\# P^{\prime \prime}-\# P^{\prime}=\# P^{\prime \prime}-p$. We have $\# P^{\prime \prime}=\# g^{-1}(P)<d^{m} \cdot \# P-1=d^{m} \cdot p-1$ as $P^{\prime \prime}$ contains at least two critical points. It follows that $q+1 \leq\left(d^{m}-1\right) p$. Furthermore

$$
\begin{aligned}
L & =d^{m} \cdot e^{-B}=d^{m} \cdot e^{-(p-3) J} e^{-A}<d^{m} \cdot e^{-(p-3) \log d^{m}} e^{-A} \\
& \leq d^{m} \cdot e^{-\log d^{m}} e^{-A}=2 \log (\sqrt{2}+1) .
\end{aligned}
$$

By the left inequality of (2.5), we have, for any $i$,

$v_{i}^{\prime}=\frac{1}{\ell\left(\gamma_{i}, \tau_{n+m}\right)}<\sum_{\beta \in W_{i}} \frac{1}{\ell_{\widehat{\mathbb{C}} \backslash P^{\prime \prime}}(\beta)}+\frac{1}{\pi}+\frac{q+1}{L}=\sum_{\beta \in W_{i}} \frac{1}{\ell_{\widehat{\mathbb{C}} \backslash P^{\prime \prime}}(\beta)}+\frac{1}{\pi}+\frac{\left(d^{m}-1\right) p}{L}$

where $W_{i}$ is the set of all simple closed geodesics on $\widehat{\mathbb{C}} \backslash P^{\prime \prime}$ homotopic to $\psi\left(\gamma_{i}\right)$ rel $P^{\prime}$, and of length (in $\widehat{\mathbb{C}} \backslash P^{\prime \prime}$ ) less than $L=d^{m} \cdot e^{-B}$.

Claim (c). Each curve $\beta$ of $W_{i}$ is homotopic rel $P^{\prime \prime}$ to some $\psi(\eta)$, for a component $\eta$ of $G^{-1}(\gamma)$ of a unique choice $\gamma \in \Gamma$. Furthermore $\eta$ is homotopic rel $Z$ to $\gamma_{i}$, and

$$
\frac{1}{\ell_{\widehat{\mathbb{C}} \backslash P^{\prime \prime}}(\beta)}=\frac{1}{\operatorname{deg}(G: \eta \rightarrow \gamma)} \frac{1}{\ell\left(\gamma, \tau_{n}\right)} .
$$

Also the map $\beta \mapsto \eta$ is injective. 
Proof. Let $\beta \in W_{i}$. It has length in $\widehat{\mathbb{C}} \backslash P^{\prime \prime}$ less than $d^{m} \cdot e^{-B}$ which is less than $d^{m} \cdot e^{-b}$. By Claim (b), it is homotopic rel $P^{\prime \prime}$ to a component $\psi(\eta)$ of $g^{-1}(\phi(\gamma))=\psi\left(G^{-1}(\gamma)\right)$ for a unique choice of $\gamma \in \Gamma$. But $\gamma$ being nonperipheral rel $Z$, the curves in $G^{-1}(\gamma)$ are pairwise non-homotopic rel $G^{-1}(Z)$. Thus the curves in $\psi\left(G^{-1}(\gamma)\right)$ are pairwise non-homotopic rel $P^{\prime \prime}$. This shows that $\eta$ is unique. As $\beta$ and $\psi(\eta)$ are homotopic rel $P^{\prime \prime}$, they are also homotopic rel $P^{\prime}$. But $\beta$ is homotopic rel $P^{\prime}$ to $\psi\left(\gamma_{i}\right)$ by the definition of $W_{i}$. We conclude that $\psi(\eta)$ is also homotopic rel $P^{\prime}$ to $\psi\left(\gamma_{i}\right)$.

As $g: \widehat{\mathbb{C}} \backslash P^{\prime \prime} \rightarrow \widehat{\mathbb{C}} \backslash P$ is a holomorphic covering, the curve $g(\beta)$ is the simple closed geodesic of $\widehat{\mathbb{C}} \backslash P$ homotopic to $\phi(\gamma)$ rel $P$. So

$$
\ell_{\widehat{\mathbb{C}} \backslash P^{\prime \prime}}(\beta)=\operatorname{deg}(g: \beta \rightarrow g(\beta)) \cdot \ell_{\widehat{\mathbb{C}} \backslash P}(g(\beta))=\operatorname{deg}(G: \eta \rightarrow \gamma) \cdot \ell\left(\gamma, \tau_{n}\right) .
$$

The injectivity of $\beta \mapsto \eta$ follows from the fact that every curve in $\psi\left(G^{-1}(\Gamma)\right)$ is homotopic rel $P^{\prime \prime}$ to a unique simple closed geodesic of $\widehat{\mathbb{C}} \backslash P^{\prime \prime}$. This proves the claim.

It follows from this claim that

$$
\sum_{\beta \in W_{i}} \frac{1}{\ell_{\widehat{\mathbb{C}} \backslash P^{\prime \prime}}(\beta)} \leq \sum_{\gamma \in \Gamma}\left(\sum_{\eta \sim Z \gamma_{i}} \frac{1}{\operatorname{deg}(G: \eta \rightarrow \gamma)}\right) \frac{1}{\ell\left(\gamma, \tau_{n}\right)}=\left(G_{\Gamma} v\right)_{i}
$$

where the sum is taken over all curves in $G^{-1}(\gamma)$ homotopic to $\gamma_{i}$ rel $Z$, and the right equality is due to the definition of the transition matrix. It follows from (2.9) that for any $i$,

$$
v_{i}^{\prime} \leq\left(G_{\Gamma} v\right)_{i}+\frac{1}{\pi}+\frac{\left(d^{m}-1\right) p}{L} .
$$

Therefore

$$
\left|v^{\prime}\right| \leq\left|G_{\Gamma} v\right|+\frac{1}{\pi}+\frac{\left(d^{m}-1\right) p}{L} \leq\left\|G_{\Gamma}\right\| \cdot|v|+\frac{1}{\pi}+\frac{\left(d^{m}-1\right) p}{L},
$$

where $|v|$ denotes the sup norm of $\mathbb{R}^{\Gamma}$. As the multicurve $\Gamma$ is $(F, Z)$-stable, we have $G_{\Gamma}=\left(F_{\Gamma}\right)^{m}$. By the choice of $m$, we have $\left\|G_{\Gamma}\right\| \leq \frac{1}{2}$. Thus

$$
\left|v^{\prime}\right| \leq \frac{1}{2}|v|+\frac{1}{\pi}+\frac{\left(d^{m}-1\right) p}{L} .
$$

If

$$
|v|>2\left(\frac{1}{\pi}+\frac{\left(d^{m}-1\right) p}{L}\right),
$$

then $\left|v^{\prime}\right|<|v|$, that is, $w\left(\tau_{n+m}\right)<w\left(\tau_{n}\right)$. Now we see that if we choose

$$
C=\max \left\{\log \left(\frac{1}{\pi}+\frac{\left(d^{m}-1\right) p}{L}\right)+\log 2, B\right\},
$$


then the proposition is proved.

\section{Applications of Thurston's theorem and related results}

\subsection{Geyer's sharpness result for harmonic polynomials}

The power of Thurston's theorem is beautifully illustrated by a result of L. Geyer. We present this result here.

Let $\pi$ denote the map $z \mapsto \bar{z}$. We say that $P(z)=a_{d} z^{d}+\cdots+a_{1} z+a_{0}$ is a Geyer polynomial if $P$ has all coefficients real, all critical points simple, at most one critical point real, and maps each critical point $c$ to its complex conjugate $\bar{c}$.

Theorem 3.1 (Geyer, $[\mathrm{Ge}])$. For every $d \geq 2$, there is a Geyer polynomial $P$ of degree $d$.

This result solved a sharpness problem in the study of harmonic polynomials. It has been conjectured by Wilmshurst ([Wil]) that for any polynomial $P$ of degree $d \geq 2$, the equation

$$
P(z)=\bar{z}
$$

has at most $3 d-2$ solutions. Khavinson and Świątek ([KS]) proved the conjecture and showed that for $d=2,3$ there are polynomials realizing the bound. Then Crofoot and Sarason noticed that the bound $3 d-2$ would be realized by a Geyer polynomial of degree $d$ if it exists. Later on Bshouty and Lyzzaik proved that such polynomials exist for $d=4,5,6$ and 8 ([BL]). But their method seems to be difficult to reach the remaining degrees.

Proof of Theorem 3.1. The idea is to first construct a topological model, and then prove the existence using Thurston's theorem.

Fix any $d \geq 2$. Assume that there exists a branched covering of $\widehat{\mathbb{C}}$ of degree $d$ satisfying $G^{-1}(\infty)=\infty, G \circ \pi=\pi \circ G$, all critical points are simple, at most one critical point is real, and each critical point $c$ is mapped to its complex conjugate $\bar{c}$. (Please refer to Geyer, [Ge] for a construction). The postcritical set of $G$ coincides with the set of critical points $\mathcal{C}_{G}$. Set $Z=\mathcal{C}_{G}$.

Let $\pi: z \mapsto \bar{z}$.

Notice that all critical points of $G$ are periodic (of period 1 or 2 ). A theorem of S. Levy proves that in this case $(G, Z)$ has no Thurston obstructions. Furthermore, fix a non-real critical point $c$ of $G$. Let $\left(\phi_{n}, f_{n}\right)$ be the sequence in Thurston's algorithm (2.1) so that every $\phi_{n}$ fixes pointwise $\infty, c, \bar{c}$, and $\phi_{0}=\pi \circ \phi_{0} \circ \pi^{-1}$.

It follows from Theorem 2.2 that $f_{n}$ converges uniformly to a polynomial $P$ combinatorially equivalent to $G$. 
We want to prove that $P$ is real. For this we will show that $f_{n}$ is real for every $n$.

Set $\phi_{1}^{\prime}=\pi \circ \phi_{1} \circ \pi^{-1}$ and $F=\pi \circ f_{1} \circ \pi^{-1}$. Then $F(z)=\overline{f_{1}(\bar{z})}$ is again a polynomial and we have the following chains of commutative diagrams:

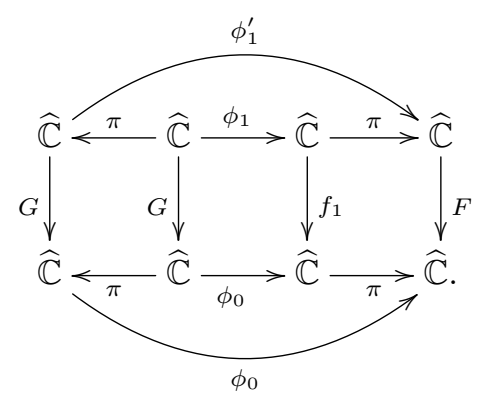

Due the uniqueness of the normalized $\psi$ making $\phi_{0} \circ G \circ \psi^{-1}$ holomorphic, we conclude that $\pi \circ \phi_{1} \circ \pi=\phi_{1}^{\prime}=\phi_{1}$. So $\phi_{1}$ is real. This in turn implies that $f_{1}$ is real.

$$
\begin{aligned}
& \text { So } \phi_{0} \text { real } \Longrightarrow\left\{\begin{array} { c } 
{ \phi _ { 1 } } \\
{ f _ { 1 } }
\end{array} \text { real } \Longrightarrow \left\{\begin{array}{l}
\phi_{n} \\
f_{n}
\end{array} \text { real } .\right.\right. \\
& \text { But } f_{n} \rightarrow P, \text { so } P \text { is real. }
\end{aligned}
$$

\subsection{Applications of Thurston's theorem}

There are many applications of Thurston's theorem in holomorphic dynamics. In most cases, there is no need to work directly with Teichmüller spaces. One just need to study Thurston obstructions.

As illustrated by Geyer's result above, the general procedure of an application goes as follows:

a. Construct a postcritically finite branched covering $F$ with some specific dynamical properties (if possible).

b. Check whether $F$ has Thurston obstructions.

c. In the case of absence of obstructions use Thurston's theorem to get a (unique up to Möbius conjugation) rational map $f$ combinatorially equivalent to $F$, therefore having the same dynamical properties.

Here is a case where there is an obstruction of topological nature: there is no branched covering of degree 4 having one double critical point $c$, four simple critical points sharing two critical values $v$ and $w$. To prove it by contradiction, draw a segment linking $v$ to $w$ through the critical value coming from $c$, and pullback this segment. One runs easily into trouble due to Jordan curve theorem. 
Another interesting case is that although it is easy to construct a cubic branched covering $F$ with 4 distinct and fixed critical points, no cubic rational map has this property. So such a $F$ must have a Thurston obstruction.

It is in general difficult to apply Thurston's theorem effectively, namely to check whether a specific branched covering has Thurston obstructions or not. Each successful application is usually a theorem in its own right. Here is brief account of some related results:

- Topological polynomials. These are the branched coverings of $S^{2}$ with one backward invariant point. S. Levy ([Levy, Go]) reduced Thurston's obstructions to some specific type of obstructions (called the Levy cycles). An easy consequence is that if every critical point eventually lands in a periodic cycle containing a critical point, then the map is unobstructed. In this case the map is combinatorially equivalent to a polynomial.

- Matings of two polynomials. This is a surgery procedure in order to obtain rational maps whose Julia set is the gluing of two postcritically finite polynomial (therefore simpler) Julia sets. Obstructions often occur. Via the works of Milnor, Rees, Sharland, Shishikura, Tan, among others, some families of maps have been well understood. They include quadratic rational maps and Newton's method of cubic polynomials. See for example [Mil, Re1, Sha, Shi2, Ta1, Ta2, ST]. One may consult the beautiful animations in the webpage of Chéritat [C], as well as the article of popularization [Ta3]. It has been known that two pairs of polynomials may lead to the same rational map. An amazing recent work of Rees shows that the number of pairs giving the same rational map can be arbitrarily large [Re5]. There are also results on matings of postcritically infinite polynomials (see for example [AY, HT, YZ]).

- Captures. This is a surgery procedure to deform a polynomial so that the point at $\infty$ glides along a certain path and gets 'captured' by a bounded orbit. Again obstructions may occur and the procedure is highly noninjective. See the works of Wittner, Rees ([Wit,Re2-Re6]), among others.

- Blowing up an arc surgery. This consists of cutting open an invariant arc of a rational map in order to create a rational map of higher degree. This has been used in the works of Pilgrim and Tan [PT, Pi1] to construct a variety of rational maps with interesting dynamical properties - Fatou component boundaries which are homeomorphic to a figure-8, symmetries, Sierpinski carpet Julia sets, maps with cylinders, etc.

- Classifications of a family of rational maps. This consists of studying a full set of combinatorics that arises in a given family. Such combinatorics may take the form of Hubbard trees, external rays, spiders, kneading sequences, laminations, graphs, etc. See for example the works of Bielefield, Geyer, Hubbard, Kiwi, Mikulich, Poirier, Rees, Rückert, Schleicher 
([BFH, G, HS, Ki, Mik, MR, Po, Re2-Re6]), among others. See also Douady-Hubbard-Sullivan's proof of the monotonicity of the topological entropy in the logistic family presented by Milnor and Thurston in [MT].

- Criteria of absence of Thurston's obstructions. Several techniques have been developed in various situations. See for example work of Bonnot, Braverman, Pilgrim, Shishikura, Tan and Yampolsky [BBY, Pi3, PT, Shi3, ST].

- Perron algebraic number as the exponential of the topological entropy. Thurston ([Th3]) proved recently that any positive algebraic number greater than the modulus of its Galois conjugates can be realized as the leading eigenvalue of a transition matrix associated to a polynomial action on its Hubbard tree.

- Folding surgery. This is a new type of surgery providing examples of postcritically finite rational maps whose Julia set contains wandering separating continua, see [CT2]. It is known, due to works of Thurston, Kiwi and Levin, [Th3, Ki, Levin], that such continua do not exist for polynomials with locally connected Julia sets (in particular for postcritically finite polynomials).

- N. Selinger studies compactifications of rational map Teichmüller spaces, [Se]. Work of Bonk, Haïssinsky, Meyer and Pilgrim, [BM, HP1, HP2, HP3, HP4, Me1, Me2, Pi2, Pi3] study postcritically finite branched coverings of $S^{2}$, in particular those with Thurston obstructions. RiveraLetelier, [Ri], studies some weakly hyperbolic rational maps with the help of the convergence of Thurston's algorithm.

- Bisets as algebraic invariant of combinatorial equivalent classes.

Let $f$ be a postcritically finite rational map. Let $t \notin \mathcal{P}_{f}$. Set $G=$ $\pi_{1}\left(\widehat{\mathbb{C}} \backslash \mathcal{P}_{f}, t\right)$.

Define $\mathfrak{M}_{f}$ to be the set of homotopic paths in $\widehat{\mathbb{C}} \backslash \mathcal{P}_{f}$, linking $t$ to a point in $f^{-1}(t)$. This set is equipped with a right action of $G$ by amending a curve $\delta \in G$ first before taking $\gamma \in \mathfrak{M}_{f}$ to get $\gamma . \delta$, and with a left action of $G$ by taking $\gamma \in \mathfrak{M}_{f}$ first and then by following the corresponding lift by $f$ of $\delta \in G$. These two actions commute and make $\mathfrak{M}_{f}$ into a $G$-biset.

Nekrachevych introduced this notion and proved that $\mathfrak{M}_{f}$ is a complete invariant of the combinatorial equivalence class of $f([\mathrm{~N} 1])$. L. Bartholdi and V. Nekrachevych then used this invariant to solve the so-called twisted rabbit problem of Hubbard, [BN1]. See also their related works as well as that of K. Bux, G. Kelsey and R. Perez [BN2, Ke, N2, N3], among others.

- Extensions of Thurston's theorem beyond postcritically finite maps. 
Thurston's original theorem can only be applied to postcritically finite rational maps. On the one hand, all these maps have a connected Julia set; on the other hand, they form a totally disconnected subset in the parameter space (except for the Lattès examples). Therefore the theorem alone cannot characterize the combinatorics of disconnected Julia sets, nor the dynamical bifurcations through continuous parameter perturbations.

Up to now there are several extensions of Thurston's theory to postcritically infinite rational maps. David Brown [Br], supported by previous work of Hubbard and Schleicher [HS], has extended it to uni-critical polynomials with an infinite postcritical set (but always with a connected Julia set), and pushed it even further to the infinite degree case, namely the exponential maps. Hubbard-Schleicher-Shishikura [HSS] extended Thurston's theorem to postcritically finite exponential maps. Zhang announced a corresponding result for maps that have a fixed Siegel disc with bounded type rotation number and are postcritically finite elsewhere. Jiang-Zhang [JZ], in parallel with Cui-Tan [CT1] solved the characterization problem for sub-hyperbolic rational maps with possibly disconnected Julia set. The proof of the former uses similar ideas as Thurston's. Whereas that of the latter reduces the situation to a postcritically finite setting and applies the marked Thurston's theorem (the unmarked one is not enough for this purpose), and at the same time provides a combination result together with a detailed description of the structure of disconnected Julia sets, alongside a Thurston-like theory for maps that are only partially defined.

G. Zhang, [Z], has generalized Thurston's theorem to maps with a fixed Siegel disc of bounded rotation number (and postcritically finite elsewhere). A generalization to maps with parabolic periodic points is also under preparation ([CT3]).

X. Wang, [Wa], developed a Thurston-like theory for rational maps with Herman rings and Siegel disks, by combining the work of [CT1] and [Z] together with a surgery technique of Shishikura [Shi1].

- Covering properties of Thurston's pullback maps.

Let $f: \widehat{\mathbb{C}} \rightarrow \widehat{\mathbb{C}}$ be a postcritically finite rational map with postcritical set $\mathcal{P}_{f}$. It induces a Thurston's pullback map $\sigma_{f}: \mathcal{T}_{\mathcal{P}_{f}} \rightarrow \mathcal{T}_{\mathcal{P}_{f}}$ which has a unique fixed point $\circledast=[\mathrm{id}] \in \mathcal{T}_{\mathcal{P}_{f}}$. For any $\tau \in \mathcal{T}_{\mathcal{P}_{f}}$, the sequence $\left(\sigma_{f}^{n}(\tau)\right)_{n \geq 0}$ converges to $\circledast$ as $n \rightarrow+\infty$.

We mention here a result about the covering properties of $\sigma_{f}$.

Theorem 3.2 (Buff-Epstein-Koch-Pilgrim,[BEKP]). (1) Assume $f$ is a polynomial of degree $\geq 2$ whose critical points are all periodic. 
Then $\sigma_{f}\left(\mathcal{T}_{\mathcal{P}_{f}}\right)$ is open and dense in $\mathcal{T}_{\mathcal{P}_{f}}$ and $\sigma_{f}: \mathcal{T}_{\mathcal{P}_{f}} \rightarrow \sigma_{f}\left(\mathcal{T}_{\mathcal{P}_{f}}\right)$ is a covering map. In particular the derivative of $\sigma_{f}$ at $\circledast$ is invertible.

(2) The rational map $f(z)=\frac{3 z^{2}}{2 z^{3}+1}$ is postcritically finite. The associated Thurston's pullback map $\sigma_{f}: \mathcal{T}_{\mathcal{P}_{f}} \rightarrow \mathcal{T}_{\mathcal{P}_{f}}$ is a ramified covering whose group of deck transformations acts transitively on the fibers, and the derivative of $\sigma_{f}$ at $\circledast$ is not invertible.

(3) There are explicit postcritically finite polynomials and rational maps $f$ for which $\sigma_{f}: \mathcal{T}_{\mathcal{P}_{f}} \rightarrow \mathcal{T}_{\mathcal{P}_{f}}$ is constant. For example, this is the case for the polynomial

$$
f(z)=2 i\left(z^{2}-\frac{1+i}{2}\right)^{2} .
$$

\section{Epstein's transversality results}

From now on, we assume that

- $f: \widehat{\mathbb{C}} \rightarrow \widehat{\mathbb{C}}$ is a rational map,

- $X$ and $Y$ are finite subsets of $\widehat{\mathbb{C}}$ containing at least three points with $\mathcal{V}_{f} \subseteq Y$ and $X \subseteq Y \cap f^{-1} Y$, and

- either $f$ is not a $(2,2,2,2)$-map or $X$ does not contain the entire postcritical set $\mathcal{P}_{f}$.

In Section 1.4, we used Thurston's contraction principle, i.e., the injectivity of the operator $\nabla_{f}=\mathrm{id}-f_{*}$ acting on the space of meromorphic quadratic differentials on $\widehat{\mathbb{C}}$ having at most simple poles, to show the smoothness of the deformation space $\operatorname{Def}_{X}^{Y}(f)$. In addition, let $\circledast$ stand for the basepoint in $\operatorname{Def}_{X}^{Y}(f)$ represented by the triple (id,id, $f$ ) as in (1.5). Then, the proof shows that the cotangent space to $\operatorname{Def}_{X}^{Y}(f)$ at $\circledast$ is canonically identified with the quotient space $\mathcal{Q}(Y) / \nabla_{f} \mathcal{Q}(X)$.

Right after his Ph.D. thesis, Epstein observed that he could deduce corresponding results for appropriate loci of maps with given multipliers, parabolic degeneracies, and holomorphic indices, from the injectivity of $\nabla_{f}$ on appropriate spaces of meromorphic quadratic differentials with higher order poles. The reader who is not a dynamicist is invited to focus on the statements related to the multipliers, since we think those are the most easily accessible ones.

\subsection{Formal invariants of a cycle}

Let us recall the following classical definitions. A point $x \in \widehat{\mathbb{C}}$ is a periodic point of $f$ of period $p$ if $f^{\circ p}(x)=x$ for some least integer $p \geq 1$. The multiplier 
$\rho$ of the cycle

$$
\langle x\rangle=\left\{x, f(x), \ldots, f^{\circ(p-1)}(x)\right\}
$$

is the eigenvalue of the derivative $D_{x}\left(f^{\circ p}\right): T_{x} \widehat{\mathbb{C}} \rightarrow T_{x} \widehat{\mathbb{C}}$. The cycle is

- superattracting if $\rho=0$,

- attracting if $0<|\rho|<1$,

- repelling if $|\rho|>1$,

- irrationally indifferent if $|\rho|=1$ and $\rho$ is not root of unity, and

- parabolic if $\rho$ is a root of unity.

The holomorphic index of $f$ along $\langle x\rangle$ is the residue

$$
\iota=\operatorname{Res}_{x} \frac{\mathrm{d} \zeta}{\zeta-\zeta \circ f^{\circ p}}
$$

where $\zeta$ is a local coordinate at $x$. It is remarkable that this residue does not depend on the choice of local coordinate $\zeta$. If $\rho \neq 1$, then

$$
\iota=\frac{1}{1-\rho} \text {. }
$$

When $\rho=\mathrm{e}^{2 \pi \mathrm{i} r / s}$ is a $s$-th root of unity, there are

- a unique integer $m \geq 1$ called the parabolic multiplicity of $f^{\circ p}$ at $x$,

- a unique complex number $\beta \in \mathbb{C}$ called the résidu itératif of $f$ at $x$ and

- a (non unique) local coordinate $\zeta$ vanishing at $x$

such that the expression of $f$ is

$$
\zeta \mapsto \rho \zeta\left(1+\zeta^{m s}+\left(\frac{m s+1}{2}-\beta\right) \zeta^{2 m s}\right)+\mathcal{O}\left(\zeta^{2 m s+2}\right) .
$$

Such a coordinate $\zeta$ is called a preferred coordinate for $f$ at $x$. The résidu itératif $\beta$ of $f$ at $x$ is related to the holomorphic index $\iota$ of $f^{\circ s}$ at $x$ by

$$
\iota=\frac{m s+1}{2}-\frac{\beta}{s}
$$

(see for example Buff-Epstein, [BE]).

Let us now assume that $x \in U$ is a periodic point of $f$ of period $p$ and let $\langle x\rangle$ be the cycle containing $x$. The formal invariants of the cycle are by definition the formal invariant of $f^{\circ p}$ at any point of the cycle (they do not depend on the point of the cycle). 


\subsection{Quadratic differentials with higher order poles}

We shall say that two quadratic differentials $q_{1}$ and $q_{2}$ which are defined and meromorphic in a neighborhood of a point $z \in \widehat{\mathbb{C}}$ represent the same divergence at $z$ if $q_{1}-q_{2}$ has at most a simple pole $z$. We shall denote by $\mathcal{D}_{z}$ the vector space of divergences $[q]_{z}$ at $z$.

For $(f, X, Y)$ as above, let $C \subseteq X$ be a union of cycles of $f$ contained in $X$. Denote by $\mathcal{D}_{C}$ the direct sum

$$
\mathcal{D}_{C}=\bigoplus_{z \in C} \mathcal{D}_{z} .
$$

In other words, a divergence at $z$ is a polar part of degree $\leq-2$ of meromorphic quadratic differentials at $z$.

We shall denote by $\widehat{\mathcal{Q}}_{C}(X)$ (respectively $\widehat{\mathcal{Q}}_{C}(Y)$ ) the set of meromorphic quadratic differentials on $\widehat{\mathbb{C}}$ which are holomorphic outside $X$ (respectively $Y$ ) and have at most simple poles outside $C$. Note that $\mathcal{Q}(X) \subset \widehat{\mathcal{Q}}_{C}(X)$ and $\mathcal{Q}(Y) \subset \widehat{\mathcal{Q}}_{C}(Y)$ and moreover, we have the canonical identifications

$$
\widehat{\mathcal{Q}}_{C}(X) / \mathcal{Q}(X) \simeq \widehat{\mathcal{Q}}_{C}(Y) / \mathcal{Q}(Y) \simeq \mathcal{D}_{C}
$$

In addition, the linear operator $\nabla_{f}$ descends to the quotient space (we keep the notation $\nabla_{f}$ for the induced map) and we have the following commutative diagram with exact columns and rows: Thus, the following diagram commutes:

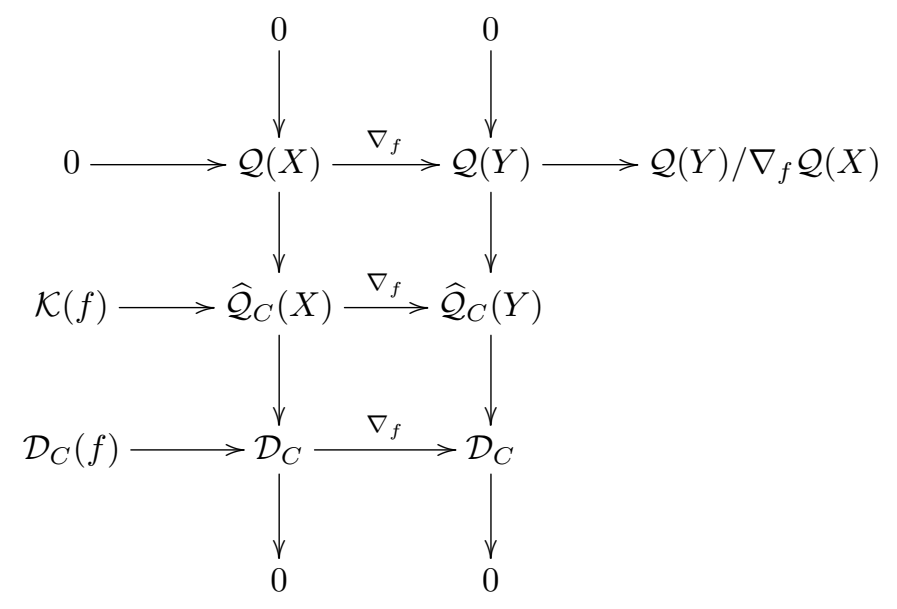

where $\mathcal{K}(f)$ is the kernel of the linear map $\nabla_{f}: \widehat{\mathcal{Q}}_{C}(X) \rightarrow \widehat{\mathcal{Q}}_{C}(Y)$ and $\mathcal{D}_{C}(f)$ is the kernel of the linear map $\nabla_{f}: \mathcal{D}_{C} \rightarrow \mathcal{D}_{C}$. 


\subsection{The Fatou-Shishikura inequality}

According to the Snake Lemma, there is a linear map $\boldsymbol{\nabla}_{f}: \mathcal{D}_{C}(f) \rightarrow \mathcal{Q}(Y) / \nabla_{f} \mathcal{Q}(X)$ such that the following sequence is exact:

$$
0 \rightarrow \mathcal{K}(f) \rightarrow \mathcal{D}_{C}(f) \stackrel{\mathbf{v}_{f}}{\rightarrow} \mathcal{Q}(Y) / \nabla_{f} \mathcal{Q}(X) .
$$

Adam Epstein then gave a complete description of $\mathcal{D}_{C}(f)$. And, by analyzing $\mathcal{K}(f)$, he proved that $\boldsymbol{\nabla}_{f}$ is injective on a certain subspace of $\mathcal{D}_{C}(f)$ (the space $\mathcal{D}_{C}^{b}(f)$ defined below).

Proposition 4.1 (Epstein [E1]).

- The space $\mathcal{D}_{C}(f)$ is computed cycle by cycle:

$$
\mathcal{D}_{C}(f)=\bigoplus_{\langle x\rangle \subseteq C} \mathcal{D}_{\langle x\rangle}(f) .
$$

Let $x \in \widehat{\mathbb{C}}$ be a periodic point of $f$ of period $p$.

(1) The projection $\mathcal{D}_{\langle x\rangle} \rightarrow \mathcal{D}_{x}$ restricts to an isomorphism $\mathcal{D}_{\langle x\rangle}(f) \rightarrow \mathcal{D}_{x}\left(f^{\circ p}\right)$ whose inverse is

$$
\triangleleft_{x}: \mathcal{D}_{x}\left(f^{\circ p}\right) \stackrel{\approx}{\longrightarrow} \mathcal{D}_{\langle x\rangle}(f), \quad[q]_{x} \mapsto \bigoplus_{k=0}^{p-1}\left[f_{*}^{\circ k} q\right]_{f^{\circ k}(x)} .
$$

(2) If $\langle x\rangle$ is superattracting, then $\mathcal{D}_{x}\left(f^{\circ p}\right)=0$.

(3) If $\langle x\rangle$ is attracting, repelling or irrationally indifferent, then $\mathcal{D}_{x}\left(f^{\circ p}\right)$ is the one-dimensional vector space spanned by $\left[\frac{\mathrm{d} \zeta^{2}}{\zeta^{2}}\right]_{x}$ for any local coordinate $\zeta$ vanishing at $x$.

(4) If $\langle x\rangle$ is parabolic with multiplier $\mathrm{e}^{2 \pi \mathrm{i} r / s}$, parabolic multiplicity $m$ and résidu itératif $\beta$, then $\mathcal{D}_{x}\left(f^{\circ p}\right)$ is the direct sum of the $m$-dimensional vector space $\mathcal{D}_{x}^{m}\left(f^{\circ p}\right)$ spanned by

$$
\left[\frac{\mathrm{d} \zeta^{2}}{\zeta^{2}}\right]_{x}, \ldots,\left[\frac{\mathrm{d} \zeta^{2}}{\zeta^{s k+2}}\right]_{x}, \ldots,\left[\frac{\mathrm{d} \zeta^{2}}{\zeta^{(m-1) s+2}}\right]_{x}
$$

together with the one-dimensional vector space spanned by

$$
\left[\frac{\mathrm{d} \zeta^{2}}{\left(\zeta^{m s+1}-\beta \zeta^{2 m s+1}\right)^{2}}\right]_{x}
$$

for any preferred coordinate $\zeta$ for $f^{\circ p}$ at $x$.

Let us now introduce the subspace $\mathcal{D}_{C}^{b}(f) \subseteq \mathcal{D}_{C}(f)$ defined by

$$
\mathcal{D}_{C}^{b}(f)=\bigoplus_{\langle x\rangle \subseteq C} \mathcal{D}_{\langle x\rangle}^{b}(f)
$$


with:

- $\mathcal{D}_{\langle x\rangle}^{b}(f)=\{0\}$ if $\langle x\rangle$ is superattracting or repelling,

- $\mathcal{D}_{\langle x\rangle}^{b}(f)=\mathcal{D}_{\langle x\rangle}(f)$ if $\langle x\rangle$ is attracting or rationally indifferent or parabolic with $\Re(\beta) \leq 0$ and

- $\mathcal{D}_{\langle x\rangle}^{b}(f)=\triangleleft_{x}\left(\mathcal{D}_{x}^{m}\left(f^{\circ p}\right)\right)$ if $\langle x\rangle$ is parabolic with $\Re(\beta)>0$.

Proposition 4.2 (Epstein [E1]). The restriction

$$
\left.\boldsymbol{\nabla}_{f}\right|_{\mathcal{D}_{C}^{b}(f)}: \mathcal{D}_{C}^{b}(f) \rightarrow \mathcal{Q}(Y) / \nabla_{f} \mathcal{Q}(X)
$$

is injective.

As an immediate corollary, Epstein refined the Fatou-Shishikura inequality on the number of non-repelling cycles of a rational map. The non refined version is the following. The proof we present is due to Epstein.

Theorem 4.3 (Shishikura, [Shi1]). A rational map of degree $d \geq 2$ has at most $2 d-2$ non-repelling cycles.

Proof. If $f$ is a $(2,2,2,2)$-map, then all the cycles are repelling. Otherwise, let $C_{0}$ be the union of superattracting cycles of $f$ and let $C$ be a union of cycles of $f$ which are non-repelling and non-superattracting. Let $X \subset \widehat{\mathbb{C}}$ be the union of $C_{0} \cup C$ with, if necessary, a repelling cycle of $f$ so that $|X| \geq 3$. Set $Y=\mathcal{V}_{f} \cup X$.

$$
\begin{aligned}
& \#\{\langle x\rangle \subseteq C,\langle x\rangle \text { non repelling }\} \\
\leq & \sum_{\langle x\rangle \subseteq C} \operatorname{dim} \mathcal{D}_{\langle x\rangle}^{b}(f) \quad \text { since each cycle contributes } \geq 1 \text { dimension } \\
\leq & \operatorname{dim} \mathcal{D}_{C}^{b}(f) \\
\leq & \operatorname{dim} \mathcal{Q}(Y) / \nabla_{f} \mathcal{Q}(X) \quad \text { by Prop. } 4.2 \\
= & \operatorname{dim} \mathcal{Q}(Y)-\operatorname{dim} \nabla_{f} \mathcal{Q}(X) \\
= & \operatorname{dim} \mathcal{Q}(Y)-\operatorname{dim} \mathcal{Q}(X) \quad \text { due to the injectivity of } \nabla_{f}(\text { Cor. } 1.7) \\
= & (\# Y-3)-(\# X-3)=\#(Y-X) \quad \text { since } X \subseteq Y \\
\leq & \#\left(\mathcal{V}_{f} \backslash C_{0}\right) .
\end{aligned}
$$

Since each superattracting cycle contains at least one critical value of $f$, the number of non repelling cycles contained in $C_{0} \cup C$ is therefore bounded from above by $\# \mathcal{V}_{f}$, which in turn is bounded from above by $\# \mathcal{C}_{f} \leq 2 d-2$.

\subsection{Transversality for multiplier loci}

Now, recall that there is a natural map $\Phi: \operatorname{Def}_{X}^{Y}(f) \rightarrow \operatorname{Rat}_{d} / \operatorname{Aut}(\widehat{\mathbb{C}}):$ if $(\psi, \phi, g)$ is a triple representing a point $\tau \in \operatorname{Def}_{X}^{Y}(f)$ as in $(1.5)$, then the 
rational map $g$ represents $\Phi(\tau)$. If $\langle x\rangle$ is a cycle of $f$ contained in $X$, then its image $\phi\langle x\rangle=\psi\langle x\rangle$ is a cycle of $g$. Since the multiplier of a cycle is invariant under holomorphic change of variables, in particular under Möbius conjugacy, the multiplier $\rho_{\langle x\rangle}(\tau)$ of this cycle only depends on $\tau$, not on the triple representing $\tau$. This defines a multiplier function

$$
\rho_{\langle x\rangle}: \operatorname{Def}_{X}^{Y}(f) \rightarrow \mathbb{C}
$$

Theorem 4.4 (Epstein [E2]). Assume $f$ is a rational map of degree $d \geq 2$ and $\langle x\rangle$ is a non-superattracting cycle of $f$. Let $X$ and $Y$ be finite subsets of $\widehat{\mathbb{C}}$ containing at least three points such that $\langle x\rangle \subseteq X \subseteq Y \cap f^{-1} Y$ and $\mathcal{V}_{f} \subseteq Y$. Let $\circledast \in \operatorname{Def}_{X}^{Y}(f)$ be the point represented by the triple (id,id, $f$ ). Then the logarithmic derivative $D_{\circledast} \log \rho_{\langle x\rangle}: T_{\circledast} \operatorname{Def}_{X}^{Y}(f) \rightarrow \mathbb{C}$ is the cotangent vector

$$
D_{\circledast} \log \rho_{\langle x\rangle}=\nabla_{f} \circ \triangleleft_{x}\left[\frac{\mathrm{d} \zeta^{2}}{\zeta^{2}}\right]_{x} \in \mathcal{Q}(Y) / \nabla_{f} \mathcal{Q}(Y)
$$

where $\zeta$ is any local coordinate vanishing at $x$.

Finally, let $C$ be a collection of non-repelling, non-superattracting cycles of $f$. For $\langle x\rangle \subseteq C$, let $V_{\langle x\rangle}$ be the analytic subset of $\operatorname{Def}_{X}^{Y}(f)$ defined by

$$
V_{\langle x\rangle}=\left\{\tau \in \operatorname{Def}_{X}^{Y}(f) \mid \rho_{\langle x\rangle}(\tau)=\rho_{\langle x\rangle}(\circledast)\right\} .
$$

The injectivity result of Epstein (Proposition 4.2) implies that the logarithmic derivatives $\left(D_{\circledast} \log \rho_{\langle x\rangle},\langle x\rangle \subseteq C\right)$ are linearly independent. In particular, we have the following transversality result.

Proposition 4.5. Near $\circledast$ in $\operatorname{Def}_{X}^{Y}(f)$, the loci $\left(V_{\langle x\rangle}\right)_{\langle x\rangle \subseteq C}$ are smooth and transverse complex submanifolds of $\operatorname{Def}_{X}^{Y}(f)$.

The reader may be interested in transferring such a transversality result to various spaces, such as the space $\operatorname{Rat}_{d}$, or the orbifold $\operatorname{Rat}_{d} / \operatorname{Aut}(\widehat{\mathbb{C}})$, or the space of monic centered polynomials of degree $d$, or the space of rational maps of degree $d$ with marked critical points, and so on. To achieve this goal, one can try to prove that there is an immersion from $\operatorname{Def}_{X}^{Y}(f)$ to the considered space or orbifold (in the latter case, one has to be cautious with such a notion since one then has to define the tangent space to an orbifold). For an example of how to proceed, one may consult [E2] where Epstein characterizes the cases where $\Phi: \operatorname{Def}_{X}^{Y}(f) \rightarrow \operatorname{Rat}_{d} / \operatorname{Aut}(\widehat{\mathbb{C}})$ is an immersion. 


\section{Acknowledgements}

We thank Adam Epstein for the time he took to explain us his results. We are grateful to Adam Epstein and Kevin Pilgrim for their helpful comments, to Wang Xiaoguang for his careful reading, and to Athanase Papadopoulos for his encouragement in the writing of this chapter.

\section{References}

[AY] M. Aspenberg and M. Yampolsky, Michael Mating non-renormalizable quadratic polynomials. Comm. Math. Phys. 287 (2009), no. 1, 1-40.

[BN1] L. Bartholdi \& V. Nekrashevych, Thurston equivalence of topological polynomials. Acta Math. 197 (2006), no. 1, 1-51.

[BN2] L. Bartholdi \& V. Nekrashevych, Iterated monodromy groups of quadratic polynomials. I. Groups Geom. Dyn. 2 (2008), no. 3, 309-336.

[BFH] B. Bielefield, Y. Fisher and J. H. Hubbard, The classification of critically preperiodic polynomials as dynamical systems, J. Amer. Math. Soc. vol. 5 (1992), 721-762.

[BBY] S. Bonnot, M. Braverman \& M.Yampolsky, Thurston equivalence to a rational map is decidable, arXiv:1009.5713.

[BF] B. Branner \& N.Fagella, Quasiconformal Surgery in Holomorphic Dynamics, monograph in preparation.

[Br] D. Brown, Thurston equivalence without postcritical finiteness for a family of polynomial and exponential mappings, manuscript.

[BL] D. Bshouty \& A. Lyzzaik, On Crofoot-Sarason's Conjecture for Harmonic Polynomials, Computational Methods and Function Theory, Volume 4 (2004), No. 1, 35-41.

[BM] M. Bonk \& D. Meyer, Expanding Thurston Maps, arXiv:1009.3647.

[BE] X. Buff \& A.L. Epstein, A parabolic Pommerenke-Levin-Yoccoz inequality, Fund. Math. 172 (2002), 249-289.

[BEKP] X. Buff, A.L. Epstein, S. Koch \& K. Pilgrim, On Thurston's pullback map, in Complex Dynamics, Families and Friends, ed. D. Schleicher, A K Peters (2009).

[BP] K. Bux \& R. Perez, On the growth of iterated monodromy groups. Topological and asymptotic aspects of group theory, 61-76, Contemp. Math., 394, Amer. Math. Soc., Providence, RI, 2006.

[CJ] T. Chen \& Y. Jiang, Canonical Thurston Obstructions for Sub-Hyperbolic Semi-Rational Branched Coverings, arXiv:1101.2285.

[C] A. Chéritat, http://www.math.univ-toulouse.fr/cheritat/MatMovies. 
[CT1] Cui G.-Z. \& Tan L., A characterization of hyperbolic rational maps, Invent. Math., to appear.

[CT2] Cui G.-Z. \& Tan L., Foldings of polynomials, manuscript in preparation.

[CT3] Cui G.-Z. \& Tan L., Hyperbolic-parabolic deformations of rational maps, manuscript in preparation.

[DH1] A. Douady \& J. H. Hubbard, A proof of Thurston's topological characterization of rational functions, Acta Math., 171 (1993), 263-297.

[E1] A.L. Epstein, Infinitesimal Thurston Rigidity and the Fatou-Shishikura Inequality, arXiv:math/9902158v1.

[E2] A.L. Epstein, Transversality in holomorphic dynamics, manuscript available on http://www.warwick.ac.uk/mases.

[Ge] L. Geyer, Sharp bounds for the valence of certain harmonic polynomials. Proc. Amer. Math. Soc. 136 (2008), no. 2, 549-555.

[Go] S. Godillon, Construction de fractions rationnelles à dynamique prescrite, Ph.D. thesis, Université de Cergy-Pontoise, Mai 2010.

[HP1] P. Haïssinksy \& K. Pilgrim, Coarse expanding conformal dynamics, Astérisque 325, 2009.

[HP2] P. Haïssinksy \& K. Pilgrim, Thurston obstructions and Ahlfors regular conformal dimension. J. Math. Pures Appl. (9) 90 (2008), no. 3, 229-241.

[HP3] P. Haïssinksy \& K. Pilgrim, Examples of coarse expanding conformal dynamical systems, Proceedings of the conference "Dynamical Systems II, Denton 2009", special volume of Discrete and Continuous Dynamical Systems, to appear.

[HP4] P. Haïssinksy \& K. Pilgrim, Finite type coarse expanding conformal dynamics, Groups, Geometry, and Dynamics, to appear.

[HT] P. Haïssinsky \& L. Tan, Convergence of pinching deformations and matings of geometrically finite polynomials. Fund. Math. 181 (2004), no. 2, 143-188.

[Hen] C. Henriksen, The combinatorial rigidity conjecture is false for cubic polynomials, Trans. Amer. Math. Soc. vol. 355 (2003), 3625-3639.

[Hu] J. H. Hubbard, Teichmüller Theory and Applications to Geometry, Topology, and Dynamics, Volume I: Teichmüller Theory, Matrix edition, 2006.

[HS] J. H. Hubbard \& D. Schleicher, The Spider Algorithm, Complex Dynamical Systems, The Mathematics behind the Mandelbrot and Julia Sets, Proceedings of Symposia in Applied Mathematics, Vol. 49, Robert Devaney, ed., A.M.S. (1994), 155-180.

[HSS] J. H. Hubbard, D. Schleicher \& M. Shishikura, Exponential Thurston maps and limits of quadratic differentials, J. of AMS, 22 (2009), 77-117.

[JZ] Y. Jiang \& G. Zhang, Combinatorial characterization of sub-hyperbolic rational maps, Adv. in Math., vol. 221 (2009), 1990-2018. 
[Ke] G. Kelsey, Mapping schemes realizable by obstructed topological polynomials, arXiv:1005.4904.

[KS] D. Khavinson \&G. Świątek, On the number of zeros of some harmonic polynomials, Proc. Amer. Math. Soc. 131 (2003), 409-414.

[Ki] J. Kiwi, Real laminations and the topological dynamics of complex polynomials, Adv. in Math. vol. 184(2) (2004), 207-267.

[Levin] G. Levin, On backward stability of holomorphic dynamical systems, Fund. Math., 158 (1998), 97-107.

[Levy] S. Levy, Critically finite rational maps, Ph.D. Thesis, Princeton University, 1985.

[McM] C. McMullen, Complex Dynamics and Renormalization, Annals of Mathematics Studies, Princeton University Press, 1994.

[MS] C. McMullen \& D. Sullivan, Quasiconformal homeomorphisms and dynamics. III. The Teichmüller space of a holomorphic dynamical system, Adv. Math. 135 (1998), no. 2, 351-395.

[Me1] D. Meyer, Invariant Peano curves of expanding Thurston maps, arXiv:0907.1536

[Me2] D. Meyer, Expanding Thurston maps as quotients, arXiv:0910.2003

[Mil] J. Milnor, Pasting together Julia sets: a worked out example of mating. Experiment. Math. 13 (2004), no. 1, 55-92.

[MT] J. Milnor \& W. Thurston, On iterated maps of the interval, Dynamical systems (College Park, MD, 1986-87), 465-563, Lecture Notes in Math., 1342, Springer, Berlin, 1988.

[Mih] Y. Mikulich, Combinatorial classification of postcritically finite Newton maps, manuscript in preparation.

[MR] Y. Mikulich \& J. Rückert, A combinatorial classification of postcritically fixed Newton maps, manuscript in preparation.

[N1] V. Nekrashevych, Self-similar groups, Mathematical Surveys and Monographs, 117. American Mathematical Society, Providence, RI, 2005.

[N2] V. Nekrashevych, Combinatorics of polynomial iterations. Complex dynamics, 169-214, A K Peters, Wellesley, MA, 200

[N3] V. Nekrashevych, Symbolic dynamics and self-similar groups. Holomorphic dynamics and renormalization, 25-73, Fields Inst. Commun., 53, Amer. Math. Soc., Providence, RI, 2008.

[Pi1] K. Pilgrim, Cylinders for iterated rational maps, Ph.D. thesis, University of California at Berkeley, May 1994.

[Pi2] K. Pilgrim, Combinations of complex dynamical systems, Springer Lecture Notes in Mathematics No. 1827, 2003.

[Pi3] K. Pilgrim, An algebraic formulation of Thurston's characterization of rational functions, preprint. 
[PT] K. Pilgrim \& Tan L., Combining rational maps and controlling obstructions, Erg. th. and dyn. syst., vol. 18 (1998), pp. 221-246.

[Po] A. Poirier, On postcritically finite polynomials, part I, Critical portraits, Stony Brook IMS preprint \#1993/5, arxiv.org/abs/math.DS/9305207.

[Re1] M. Rees, Realization of matings of polynomials as rational maps of degree two, manuscript, 1986.

[Re2] M. Rees, A partial description of the Parameter Space of Rational Maps of Degree Two: Part 1, Acta Math. 168 (1992) 11-87.

[Re3] M. Rees, A partial description of the Parameter Space of Rational Maps of degree two: Part 2, Proc. LMS 70 (1995) 644-690.

[Re4] M. Rees, Views of parameter space: Topographer and Resident, Astérisque 288 (2003).

[Re5] M. Rees, Multiple equivalent matings with the aeroplane polynomial. Ergodic Theory and Dynamical Systems, vol. 30-04 (2010), 1239-1257.

[Re6] M. Rees, A Fundamental Domain for $V_{3}$, Mem. Soc. Math. de France, to appear.

[Ri] J. Rivera-Letelier, A connecting lemma for rational maps satisfying a nogrowth condition. Ergodic Theory Dynam. Systems, 27 (2007), no. 2, 595636.

[Se] N. Selinger, Thurston's pullback map on the augmented Teichmüller space and applications, arXiv:1010.1690.

[Sha] T. Sharland, Ph.D. thesis of Warwick University, in preparation.

[Shi1] M. Shishikura, On the quasiconformal surgery of rational functions. Ann. Sci. cole Norm. Sup. (4) 20 (1987), no. 1, 1-29.

[Shi2] M. Shishikura, On a theorem of Mary Rees for matings of polynomials, in The Mandelbrot set, Theme and Variations, ed. Tan Lei, LMS Lecture Note Series 274, Cambridge University Press, 2000, 289-305.

[Shi3] M. Shishikura, An application of Thurston's theorem on branched coverings, conference talk at the workshop: Parabolic Implosion, Toulouse, November 22, 2010

[ST] M. Shishikura \& Tan L., A family of cubic rational maps and matings of cubic polynomials, Exp. Math. 9 (2000), 29-53.

[So] D. E. K. Sørensen, Infinitely renormalizable quadratic polynomials, with non-locally connected Julia set, J. Geom. Anal. 10 (2000), no 1, 169-206.

[Su] D. Sullivan, Quasiconformal homeomorphisms and dynamics. I. Solution of the Fatou-Julia problem on wandering domains, Ann. of Math. (2) 122 (1985), no. 3, 401-418.

[Ta1] Tan L., Matings of quadratic polynomials, Erg. th. and dyn. syst., vo. 12 (1992), pp. 589-620. 
[Ta2] Tan L., Branched coverings and cubic Newton maps, Fundamenta Mathematicae, 154 (1997), pp. 207-260.

[Ta3] Tan L. La méthode de Newton et son fractal, article of vulgarization, Images des Mathématiques, CNRS, http://images.math.cnrs.fr/La-methodede-Newton-et-son.html .

[Th1] W. Thurston, The combinatorics of iterated rational maps, preprint, Princeton University, Princeton, NJ, 1983.

[Th2] W. Thurston, The combinatorics of iterated rational maps (1985), publishes in: Complex dynamics: Families and Friends, ed. D. Schleicher, A K Peters (2008), 1-108.

[Th3] W. Thurston, Entropy in dimension one, manuscript in preparation, see also: Core entropy, conference talk at the workshop on Holomorphic Dynamics around Thurstons Theorem, Roskilde, September 27-October 1, 2010 .

[Ti] V. Timorin, Topological regluing of rational functions. Invent. Math. 179 (2010), no. 3, 461-506.

[Wa] X. Wang, Thurston-type theorems for rational maps with rotation domains, manuscript in preparation.

[Wil] Wilmshurst, The valence of harmonic polynomials, Proc. Amer. Math. Soc. 126 (1998), 2077-2081.

[Wit] B. Wittner, On the bifurcation loci of rational maps of degree two, Ph.D. thesis, Cornell University, 1986.

[YZ] M. Yampolsky and S. Zakeri, Mating Siegel quadratic polynomials. $J$. Amer. Math. Soc. 14 (2001), no. 1, 25-78 (electronic).

[Z] G. Zhang, Dynamics of Siegel rational maps with prescribed combinatorics, arXiv:0811.3043. 